\title{
The Dynamics of Coarsening in Highly Anisotropic Systems: Si Particles in Al-Si Liquids
}

\author{
A. J. Shahani ${ }^{a, *}$ E. B. Gulsoy ${ }^{\mathrm{a}}$, V. J. Roussochatzakis ${ }^{\mathrm{a}}$, J. W. Gibbs ${ }^{\mathrm{a}}$, \\ J. L. Fife ${ }^{\mathrm{b}}$, P. W. Voorhees ${ }^{\mathrm{a}}$ \\ ${ }^{a}$ Department of Materials Science and Engineering, Northwestern University, Evanston, IL 60208, USA \\ ${ }^{b}$ Swiss Light Source, Paul Scherrer Institut, 5232 Villigen, Switzerland
}

\begin{abstract}
The coarsening process of an Al-29.9wt\% Si alloy is studied using four-dimensional phase contrast X-ray tomography. This alloy is composed of highly anisotropic, primary Si particles in an eutectic matrix. We analyze the morphology of the primary Si particles during coarsening by detemining the interface normal distribution and the interface shape distribution. The inverse surface area per unit volume increases with the cube root of time despite the lack of microstructural self-similarity and highly anisotropic particle morphology. More specifically, over the time frame of the experiments, the Si particles evolve from mostly faceted domains to a more isotropic structure that is not given by the Wulff shape of the crystal. These trends can be rationalized by the presence of twin defects that intersect particle edges and that may provide the kink sites necessary for interfacial propagation, thus leading to a more isotropic structure. While in many cases the interfacial velocity of Si solid-liquid interfaces is highly anisotropic, the presence of many defects leads to a highly mobile interface and diffusion-limited coarsening.
\end{abstract}

Keywords: 4D Characterization, Aluminum-Silicon Alloys, Anisotropic Growth Morphologies, Isothermal Coarsening, Phase-Contrast X-ray Tomography

\section{Introduction}

Aluminum-Silicon (Al-Si) alloys are widely used in the automobile and aerospace industries due to their low density, good strength, and excellent castability $[1,2,3]$. The microstructure of the Al-Si alloy strongly influences its mechanical properties. For instance, in cast Al-Si alloys, the eutectic is flake-like. The Si platelets act as nuclei for internal cracks, thereby reducing the fatigue strength of the alloy [4]. Similarly, hyper-eutectic Al-Si alloys consist of primary Si particles, which crystallize in anisotropic shapes, in a eutectic matrix. The morphologies of these primary Si particles have been identified as the main cause of fatigue and wear of engine parts [5]. In order to modify the microstructure of cast Al-Si alloy, many processing techniques are available:

- deliberate addition of several elements, e.g., sodium and strontium [6];

- electromagnetic stirring and ultrasonic vibrations [7];

- semi-solid metal (SSM) processing above the eutectic temperature $\left(577^{\circ} \mathrm{C}\right)[8]$.

\footnotetext{
*Corresponding author.

Email address: shahani@u.northwestern.edu (A. J. Shahani)
} 
While there have been several studies on the first two approaches (e.g., Refs. [6, 7]), the microstructural evolution of the anisotropic Si particles at high temperature is not well understood. In particular, the growth mechanism of primary Si particles, held isothermally in the solid-liquid region, needs to be investigated in order to better control the hypereutectic microstructure during SSM processing.

In this two-phase solid-liquid region, coarsening occurs, i.e., large particles grow at the expense of small particles. Coarsening decreases the energy of the system by decreasing the overall curvature of the interface between the particle and matrix [4]. The coarsening process in this highly faceted $\mathrm{Al}-\mathrm{Si}$ system, however, can be much more complex than in systems where the interfacial energy and the interfacial mobility are both isotropic. For example, while studying the growth and dissolution behavior of primary Si particles in hypereutectic Al-Si alloys during cooling and heating, respectively, Moorthy and Howe [9] found that the faster growing $\{113\}$ facets of Si grow out during cooling, leaving the slower growing $\{111\}$ facets in contact with the liquid. This indicates a high degree of anisotropy in the growth kinetics. However, they also observed that the Si solid-liquid interface is smoothly curved during dissolution, suggesting that the removal of atoms from the $\mathrm{Si}$ surface is a continuous process as opposed to occuring in abrupt jumps [9]. The effects of this change in anisotropy of the interfacial mobility on solidification and melting are discussed further in a subsequent publication [10].

It is also well known that the Si solid-liquid interfacial energy is highly anisotropic, where the $\{111\}$ planes have the lowest energy: in particular, experiments $[11,12]$ as well as molecular dynamics simulations with empirical potentials [13, 14] demonstrate that the global minimum in the surface free energy for Si occurs at the $\{111\}$ orientation. In addition, the $\{111\}$ planes have very low interfacial mobility: the Jackson $\alpha$ factor [15] for $\mathrm{Si}\{111\}$ is $2.7[16]$; the relatively high value of $\alpha\left(\alpha_{\{111\}}>2\right)$ indicates that the $\{111\}$ surface is likely atomically smooth and thus there is a barrier to nucleation of the next atomic layer $[16,15]$. Beatty and Jackson [16] simulated the growth of Si using a Monte Carlo scheme; they found that the growth rate, $v$, increases linearly with undercooling, $\Delta T$, for a rough Si $\{100\}$ plane while $v$ varies exponentially with $\Delta T$ for a $\{111\}$ facet in a $2 \mathrm{D}$ poly-nuclear growth mode $[16,17]$. For low undercooling, then, the growth velocity on a facet plane is much smaller than on a rough plane. Thus, defects can play a critical role in providing the steps necessary for interfacial propagation. One example is the twin plane re-entrant edge (TPRE) mechanism for the growth of Si dendrites in the $\{112\}$ direction $[18,19]$. Recently, Gamalski et al. [20] suggested that the presence of the twin, by itself, does not reduce the barrier for nucleation of steps; rather, the barrier is effectively reduced by the line energy.

Previous experiments $[9,21]$ have been limited to examining 2D cross-sections of the material, while most kinetic models (e.g., coarsening) make predictions based upon a 3D microstructure $[22,23,24]$. In order to assess the morphological and topological complexity of Al-Si alloy, and to analyze its evolution in time, we require a four-dimensional (i.e., 3D space plus time) analysis. X-ray tomography (XRT) is a nondestructive characterization method, where it is possible to follow the evolution of the $3 \mathrm{D}$ microstructure as a function of time $[24,25]$. In particular, X-ray phase contrast tomography (PCT), where the phase is retrieved in the near-field regime, enables the study of systems consisting of elements with similar atomic numbers, such as in an Al-Si alloy [26, 27]. Thus, we probe the microstructural evolution of primary Si particles in an Al-29.9wt\%Si alloy during isothermal coarsening via 4D PCT. Our 4D analyses provide new insights in the coarsening mechanisms in highly faceted, complex, and interconnected systems. 


\section{Methods}

\subsection{Experimental Methodology}

Al-Si rods of composition 29.9wt\%Si were prepared by Ames Laboratory [28], see Refs. $[26,27]$ for experimental details. The 4D propagation-based PCT experiment was conducted ex situ at the TOmographic Microscopy and Coherent rAdiology experimenTs (TOMCAT) beamline of the Swiss Light Source (Paul Scherrer Institut, Switzerland) [29]. The sample was placed in a custom-made isothermal furnace, and the primary Si particles were allowed to coarsen at $590^{\circ} \mathrm{C}$, just above the eutectic temperature. After 10 minutes, the sample was quenched to room temperature, and tomographic projections were collected when the sample was fully solid. The effects of quenching on the coarsening process are addressed in the discussion. Subsequently, the sample was reheated to $590^{\circ} \mathrm{C}$ for 10 minutes, continuing the coarsening of the primary Si plates. This cycle was repeated six times. Phase-retrieval and absorption-based reconstructions of the images were conducted on-site using customized algorithms based on Paganin [30] and Gridrec [31]. Each dataset was $1525 \times 1525 \times 1598 \mu \mathrm{m}^{3}$, with a voxel size of $0.74 \times 0.74 \times 0.74 \mu \mathrm{m}^{3}$.

\subsection{Data Processing}

The growing amount of data collected during XRT experiments, typically on the order of terabytes, renders manual segmentation impractical. Furthermore, processing data sets from PCT is nontrivial due to the inherently low-pass characteristics of single image phase retrieval, which results in diffuse interfaces [27, 32]. To circumvent the problems in segmenting phase contrast images, we use the integrated approach proposed by Shahani et al. [27]. In this approach, phase and absorption contrast images, obtained at a single object-detector distance, are combined linearly in real space. The resulting hybrid images feature sharper interfaces, thereby enabling the automated segmentation of such images by non-linear diffusion filtering and bias-corrected fuzzy c-means algorithm, see Ref. [27] for computational details. The 2D binary images are then combined to reveal the $3 \mathrm{D}$ volumes for quantitative microstructural characterization.

\subsection{Microstructural Analysis}

For the subsequent analyses, the digitized surfaces are meshed. Each triangular surface on the mesh and its three vertices will hereafter be referred to as a patch. To remove any meshing artifacts (e.g., staircasing) prior to analysis, we smooth the mesh by mean curvature flow [33]. Curvature flow preserves shape better than Laplacian smoothing, although it tends to shrink volume, especially for more aggressive smoothings. For this reason, we apply the least amount of curvature smoothing for each structure to maintain the integrity of the interface.

\subsubsection{Surface Area Per Unit Volume}

For infinitely separated, spherical particles undergoing coarsening, Lifshitz and Slyozov [34] and Wagner [35] proposed that the microstructure becomes self-similar when scaled by a time-dependent length scale, e.g., average particle radius. They found that the average particle radius grows with the cube-root of time for diffusion limited coarsening, and with the square-root of time for interface reaction limited coarsening $[4,34,35]$. However, since Si particles are non-spherical, we seek another time-dependent length scale to characterize the coarsening process.

One such possibility is the inverse of surface area per unit volume, $S_{v}^{-1}$, which has units of length. For instance, Marsh and Glicksman found that $S_{v}^{-1}$ was lrelated to time, 
$t$, as $t^{1 / 3}$ for the coarsening of dendritic mixtures [36]. Thus, during coarsening, the relationship between $S_{v}$ and $t$ is

$$
S_{v}^{-n}(t)-S_{v}^{-n}(0)=K t
$$

where $S_{v}(0)$ is the specific surface area at the onset of steady-state coarsening, $n=2$ for interface-limited coarsening, $n=3$ for diffusion-limited coarsening, and $K$ is a rate constant $[34,35]$. To adequately represent the kinetics of the coarsening process, we measure $S_{v}$ at each time-step. This is accomplished by summing over all patches in the mesh, and dividing by the volume $v$ of the region of interest (ROI), $296 \times 296 \times 159 \mu \mathrm{m}^{3}$,

$$
S_{v}^{T}=\frac{1}{v} \sum_{i} \frac{1}{2}\left\|\vec{e}_{i, 1} \times \vec{e}_{i, 2}\right\|
$$

where the superscript $T$ indicates that $S_{v}$ is measured from the tomography data, $i$ is the index of the triangular patch, and $\vec{e}_{i, 1}$ and $\vec{e}_{i, 2}$ are two edge vectors of patch $i$.

Due to time-constraints at the beamline, samples were only annealed for short times (less than one hour). Furthermore, the segmented ROI is only a few hundred micrometers in length. Therefore, to reinforce the findings of the tomography experiment, $S_{v}$ was calculated using optical metallography at Northwestern University, following the tomography experiment. A new as-cast sample of composition Al-29.9wt\%Si was used for the isothermal annealing experiments and stereological evaluation. Annealing times in the range of several days combined with the much larger ROIs for optical microscopy, in the millimeter range, provide reliable data to check for consistency. $S_{v}$ can be calculated from the optical micrographs via the line-intercept method $[37,38]$ : first, a grid of "test lines" is superimposed on the micrograph; next, the number of points generated per unit length of test lines, $P_{L}$, is determined. These "points" consist of intersections made by test lines with the interfaces of the Si particles. Then, $S_{v}$ is calculated as

$$
S_{v}^{M}=2 P_{L}
$$

where the superscript $M$ indicates that $S_{v}$ is measured from the optical micrographs. The above expression shows that $S_{v}$ is related to $P_{L}$ by a factor of 2 , which is due to the theoretical restriction of random orientation [38]. Thus, we measure $S_{v}^{M}$ for several time-steps over the course of two days, for an Al-29.9wt\% Si sample annealed at $600^{\circ} \mathrm{C}{ }^{1}$

\subsubsection{Interface Normal Distribution}

Despite being morphology-independent, the length scale $S_{v}^{-1}$ is an average over the system and cannot account for any localized evolution of the microstructure. In order to measure these microstructural details, we determine the evolution of the orientation of a patch of interface and the curvature of a patch of interface, with coarsening time. In particular, directionality in the microstructure can be determined by creating a stereographic projection of the interfacial patch normals. By scaling the stereographic projection with the patch area, these plots represent the probability of locating an interfacial patch with particular spatial orientation, referred to as the IND [23, 39, 40]. It is important to note that the IND is constructed in the laboratory coordinates, and not the coordinates of the

\footnotetext{
${ }^{1}$ The annealing temperature for metallography was $10^{\circ} \mathrm{C}$ higher than that for in situ tomography. The small difference in annealing temperature should only influence the measured rate constant $K$ and not the coarsening mechanism or temporal dependence given by $n$ in Eq. (1).
} 
crystal lattice. Thus, due to the independent Si particles in the structure, the IND is a superposition of many single crystal patterns.

In order to construct an IND of a given microstructure, the unit normals of each patch are determined. We adopt the thermodynamic convention for calculating interface normals, where the normals point from Si particle to liquid. The orientation of a patch of interface is specified by the point of intersection of the normal vector with the unit hypersphere [41]. These normals are then projected onto a pre-defined projection plane, tangent to the unit hypersphere, using angular relationships, see Ref. [42]. Conventionally, the upper and lower projection planes are used to fully quantify the directionality in the northern and southern hemispheres of the unit hypersphere, respectively. To avoid having to show two INDs of a given microstructure, we project the normals onto a single cylinder, tangent to the unit hypersphere at its equator. The coordinates $\left(\theta^{\prime}, \phi^{\prime}\right)$ of this Mercator projection are given by

$$
\begin{aligned}
\theta^{\prime} & =\theta-\theta_{0} \\
\phi^{\prime} & =\ln \left[\tan \left(\frac{\pi}{2}-\frac{\phi}{2}\right)\right]
\end{aligned}
$$

where $(\theta, \phi)$ are the azimuth and elevation coordinates on the unit hypersphere, respectively, and $\theta_{0}$ is an arbitrarily centered meridian. The mapping of Eq. (4) represents an angle-preserving projection although there are distortions towards the north and south poles of the unit hypersphere, i.e., as $\phi \rightarrow\{0, \pi\}$. If the normals are equatorial, however, then there is little discernable distortion in the projection. Furthermore, it should be noted that the normals are binned on the hypersphere and not on the Mercator projection to avoid any artifacts arising from the nonequal-area nature of the projection [39].

While the predominant $\{111\}$ poles in the microstructure can be indexed experimentally, for example, using electron backscatter diffraction (EBSD) or Kikuchi diffraction (BKD) [21], we identify the $\{111\}$ poles directly from the IND using the following procedure. First, it is necessary to cluster the patches that belong to the same facet. This is necessary because, unlike in a perfectly smooth interface, the $\mathrm{Si}$ facets have finite roughness due to small segmentation errors or noise in the data. This corresponds to a finite (albeit, narrow) distribution of patch orientations in the IND. Clustering is accomplished on the unit hypersphere using spherical k-means (SKM) algorithm [43]. In this non-euclidean geometry, the "distance" measured between a centroid vector, $\vec{p}$, and a patch normal vector, $\vec{n}$, is the cosine dissimilarity, $d(\vec{n}, \vec{p})$, given by

$$
d(\vec{n}, \vec{p})=1-\frac{\vec{n} \cdot \vec{p}}{\|\vec{n}\|\|\vec{p}\|}
$$

where the notation $\|*\|$ indicates the $l_{2}$ norm. Thus, SKM minimizes the objective function $\sum_{i} d\left(\vec{n}_{i}, \vec{p}_{c(i)}\right)$ over all assignments $c$ of patch normals $i$ to clusters $c(i) \in\{1, \cdots, k\}$ and over all cluster centroids $\vec{p}_{1}, \cdots, \vec{p}_{k}{ }^{2}$ For computational details, see Ref. [43]. Once cluster centroids have been determined, the next step is to compute the angles between all centroids and compare to those between $\{111\}$ planes that meet at a large angle twin boundary [44], i.e., $70.53^{\circ}, 109.47^{\circ}$, or $180^{\circ}$. In coincident site lattice (CSL) notation, the angle-axis pair $70.53^{\circ}<110>$ represents a $\Sigma 3$ grain boundary with symmetrical tilt configuration [45]. If the angle between two given cluster centroids matches any one of

\footnotetext{
${ }^{2}$ The number of clusters, $k$, is determined by visually inspecting the hypersphere.
} 
these angles, the two corresponding poles are indexed as $\{111\}$. Because this simple procedure may (by chance) match two unrelated planes in the ROI, the $\{111\}$ assignments are confirmed by visually inspecting the surfaces in the real-space reconstructions. It should be noted that this angle matching procedure is computationally tractable since there are only a few particles in the ROI. For further details, refer to Appendix A.

\subsubsection{Interface Shape Distribution}

According to the Gibbs-Thomson equation [4], for a system with isotropic interfacial energy, the equilibrium concentration of solute at an interface during coarsening, $\mathscr{C}$, is given by

$$
\mathscr{C}=\mathscr{C}_{\infty}+\Gamma H
$$

where $\mathscr{C}_{\infty}$ is the composition of solute at a flat interface, $\Gamma$ is the capillary length determined by material parameters, and $H$ is the mean interfacial curvature. Variations in $\mathscr{C}$ at interfaces create solute concentration gradients, which cause solute flux from regions of high to low concentration. If the interfacial energy is anisotropic, but with facets, the diffusion potential, $\Gamma H$, is replaced by either the weighted mean curvature [46] or the surface divergence of the capillarity vector, $\nabla_{\text {surf }} \vec{\xi}[47,48]$. In the fully faceted limit, the expression for weighted mean curvature is simplified; in this case, tractable expressions can be produced for morphological evolution, see for instance Refs. [49, 50]. Since the $\mathrm{Si}$ particles of this study are partially faceted and partially rounded, the concentration at the interface of a $\mathrm{Si}$ particle is given by one of these equations.

Mean curvature, $H$, is defined as $\frac{1}{2}\left(\kappa_{1}+\kappa_{2}\right)$ where $\kappa_{1}$ and $\kappa_{2}$ are the minimum and maximum principal curvatures of the interface, respectively. From differential geometry, $\kappa_{1}$ and $\kappa_{2}$ are the eigenvalues of the $2 \times 2$ curvature matrix, $\mathscr{H}$ [51]. Once the interfacial curvatures are obtained, an ISD can be plotted, which provides the probability of locating an interfacial patch for a given range of principal curvatures [23, 25, 52]. Then, the ISDs at different time-steps can be used to track, quantitatively, the morphological evolution of the Si particles during coarsening. For each patch in the mesh, $\kappa_{1}$ and $\kappa_{2}$ must be determined. This is accomplished by fitting each patch and its nearest neighbors to a conic section, $f(x, y)$, of the form

$$
f(x, y)=a x^{2}+b y^{2}+c x y+d x+e y+f
$$

with respect to the local coordinate system $(x, y)$ of the patch [51]. A similar procedure is performed by Rowenhorst [53]. Specifically, the local coordinate system is defined by the $x$ and $y$ coordinates in the plane of the patch, and $z$ normal to the patch, pointing towards the eutectic. In practice, we use first, second, and third order nearest neighbor patches. Fitting is done using least-squares. Then, $\mathscr{H}$ can be expressed as

$$
\mathscr{H}=\left(\begin{array}{ll}
a & c \\
c & b
\end{array}\right)
$$

from which $\kappa_{1}$ and $\kappa_{2}$ can be calculated. ${ }^{3}$ This process is repeated for all patches in the ROI (i.e., on the order of $10^{6}$ patches). Once the principal curvatures are determined, the curvature space can be visualized by constructing a bivariate histogram of $\kappa_{1}$ versus

\footnotetext{
${ }^{3}$ The eigenvalues of the $2 \times 2$ matrix $\mathscr{H}$ are, explicitly, $\kappa=\frac{1}{2}\left(a+b \pm \sqrt{(a-b)^{2}+4 c^{2}}\right)$.
} 
$\kappa_{2}[23,39]$. However, we make use of an alternative classification of curvature proposed by Koenderink and van Doorn, namely that of $S$ versus $C$ space [54], where

$$
\begin{aligned}
& S=\frac{2}{\pi} \tan ^{-1}\left(\frac{\kappa_{2}+\kappa_{1}}{\kappa_{2}-\kappa_{1}}\right) \\
& C=\sqrt{\kappa_{1}^{2}+\kappa_{2}^{2}}
\end{aligned}
$$

and $S \in[-1,1]$ and $C \in[0, \infty)$. The major advantage of this representation is that shape and length scales are decoupled: the "shape index", $S$, specifies shape independently of length scale, whereas the "curvedness", $C$, specifies the size-scale of a specific shape in units of inverse length [54]. Furthermore, whereas both gaussian and mean curvature are required to fully describe interfacial shapes, the shape index carries all relevant shape information as a single number instead of two. In addition, due to the presence of both faceted and smooth interfaces, there is no direct connection between $H$ and the interfacial composition. So, using a probability plot in $H$ - $K$ space, where $K$ is the Gaussian curvature, is not necessarily the best option. Graphically, $S$ versus $C$ can be thought of as the polar representation of $\kappa_{1}$ versus $\kappa_{2}$. In this alternative ISD, patches have a liquid cylindrical-like shape when $S=-0.5$, a saddle-like shape when $S=0$, and a solid cylindrical-like shape when $S=0.5$. However, for a planar patch, $S$ is indeterminate while $C=0$. Fig. 1 gives an illustration of the shape $(S)$ index scale divided into nine categories.

\subsection{Texture Analysis}

While the INDs described in section 2.3.2 can be used to identify, albeit deterministically, particles meeting at a $70.53^{\circ}<110>$ symmetrical tilt boundary, there may in fact be other grain boundaries, e.g., low angle twist, etc., that are undetected with this approach. Therefore, to fully characterize the grain misorientation within the Si particles, we make use of the conventional electron backscatter diffraction (EBSD) technique. EBSD mapping was carried out for the Al-29.9wt\%Si sample annealed at $600^{\circ} \mathrm{C}$ for two days.

This sample was prepared for EBSD by first grinding the surface with 14.5, 12.2, and $6.5 \mu \mathrm{m}$ sandpaper, then subsequently polishing with a diamond suspension and etching with Keller's reagent. For each measurement, the sample was tilted to about $70^{\circ}$ with respect to the electron beam. The electron microscope was operated at $30 \mathrm{keV}$ with a working distance of $6 \mathrm{~mm}$; the step size and spot size during EBSD data collection were $1.3 \mu \mathrm{m}$ and $4.5 \mathrm{~nm}$, respectively. Indexing of electron backscatter patterns was performed on-site via the integrated Oxford AZtec software. Indices were accepted when the misfit between the simulated and detected Kikuchi bands had a mean angular deviation of less than $1^{\circ}$. Then, the texture data were analyzed via the MATLAB toolbox MTEX [55], in order to generate orientation maps and misorientation distributions.

\section{Results}

\subsection{D Reconstructions}

The microstructural evolution during coarsening is shown in the 3D reconstructions in Fig. 2, first published in Ref. [27]. The light gray particles are Si and the eutectic is transparent. Qualitatively, the increase in size scale of the structure is consistent with a coarsening process and the increasingly isotropic structure can be clearly observed. While the structures grow less interconnected and more isotropic, some degree of faceting can 
still be observed at later times. This suggests the presence of $\{111\}$ facets in the solid, that do not evolve as fast as other orientations, as previously observed in Refs. [9, 16, 21, 26]. In other words, the broad faces of a given particle, suggested to be of $\{111\}$ type, appear to be growing much slower with respect to the edges of the particle, giving rise to the observed structural changes [26].

\subsection{Surface Area Per Unit Volume}

Fig. 3 shows (a) a comparison of the metallography and tomography results, as well as (b-e) optical micrographs of some of the coarsened structures and their corresponding annealing times. In order to determine the coarsening exponent and rate constant, $K$, we fit the data to Eq. (1). Slopes of $0.29 \pm 0.03$ and $0.28 \pm 0.03$ were obtained from the metallographic and tomographic datasets, respectively, which are both far closer to the $S_{v}^{-1} \propto t^{1 / 3}$ relationship of bulk diffusion than the $S_{v}^{-1} \propto t^{1 / 2}$ relationship of interfacial diffusion. Worth noting is the displacement of the tomographic results relative to the metallographic results: this is most likely due to artificial smoothing in the tomographic data during the segmentation process [27] and mesh generation. It could also be due to the fact that the annealing temperature in the metallography experiments was $10^{\circ} \mathrm{C}$ higher than in the tomography experiments. This would lead to a difference in measured absolute values of around $10 \%$, but not a difference in measured slopes. The average coarsening rate constant $K$ is $15 \pm 2 \mu \mathrm{m}^{3} / \mathrm{min}$., where the error accounts for the relative offset (standard deviation) between the tomographic and metallographic results. The volume fraction for all time steps is consistently around the expected value of $0.30 \pm$ 0.05. Errors in the measurement of $S_{v}^{T}$ from the tomography $(T)$ data are predominantly due to mis-classified voxels during the segmentation process, in comparison to a few hand-segmented images [27], while errors in $S_{v}^{M}$ from the metallography $(M)$ data are due to counting statistics during stereological analysis.

The exponent of $1 / 3$ measured from both tomography and metallography suggest that long-range diffusion is an important factor during the coarsening process. However, one should note that the relatively immobile $\{111\}$ planes, which are the broad faces of the particles, are still governed by interfacial kinetics, or more specifically, the attachment and detachment of solute. This implies that the growth of the primary Si particles during coarsening is determined principally by growth at the particle edges. This conclusion is discussed further in subsequent sections.

\subsection{Interface Normal Distributions}

Fig. 4 shows the IND for the as-cast sample $(t=0$ min.). While dendritic samples are typically characterized by smoothly varying INDs $[23,40]$, the Si facets in this study correspond to sharp peaks in the IND. This indicates a high degree of directionality in the as-cast microstructure. Poles in the IND can be indexed as $\{111\}$ using the aforementioned procedure. In Fig. 4, the four poles A-D are of $\{111\}$ type, and belong to the same connected component, denoted ABCD. "Connected component" refers to several interconnected particles, as opposed to a single, isolated structure [26]. Poles A and $\mathrm{C}$, and $\mathrm{B}$ and $\mathrm{D}$ are $180^{\circ}$ apart due to inversion symmetry in $\{111\}$. Physically, this means that these pole pairs correspond to two opposite faces of a given particle in the connected component.

We can track the evolution of Mercator poles A-D as a function of coarsening time, see Fig. 5. Fixing the range of the color bar and the range of values $\left(\theta^{\prime}, \phi^{\prime}\right)$ that are indicative of each of the four facets allows for the comparison of multiple coarsening times. In general, pole intensities decrease with time for facets A-D, relative to the total 
surface area; however, the intensities appear to decay faster for poles B and D compared to $\mathrm{A}$ and $\mathrm{C}$. This is because particle BD is smaller than particle AC and therefore has fewer $\{111\}$ patches. The decrease in pole intensity with time is consistent with the structures becoming increasingly rounded during the coarsening process.

The range of values $\left(\theta^{\prime}, \phi^{\prime}\right)$ depicted in Fig. 5 can be segmented with an appropriate threshold $\left(4 \times 10^{-4}\right)$, to give those bins in the $\left(\theta^{\prime}, \phi^{\prime}\right)$ histogram corresponding to $\{111\}$ poles A-D. Then, the interfacial patches belonging to these bins can be illuminated on the mesh, creating a so-called Specific Microstructural Location (SML) figure [22]. The SML for connected component ABCD is shown in Fig. 6, depicting the segmented $\{111\}$ patches in red. Fig. 6(a) shows two particles that meet at a $70.5^{\circ}<110>$ symmetrical tilt grain boundary in the as-cast sample. The edges of both particles evolve much faster than the broad $\{111\}$ faces during coarsening.

The ratio of the area of these $\{111\}$ red patches in connected component ABCD, $A_{\{111\}}$, to the total surface area of the ROI, $S$, is plotted versus time in Fig. 7 . The plot shows a decrease in fractional area, $A_{\{111\}} / S$, with time, indicating that $A_{\{111\}}$ decays faster than $S .{ }^{4}$ This suggests that the Si particles are evolving away from their equilibrium Wulff shape during the first $50 \mathrm{~min}$. of coarsening. Hypothetically, a single crystal of Si coarsening near equilibrium would change volume but still preserve aspect ratio, or in this case, $A_{\{111\}} / S$ [4]. However, the complex, interconnected structures of this study are not evolving through a series of equilibrium shapes, and thus the surfaces of primary Si do not have constant chemical potential with respect to the liquid phase. Thus the mechanism for the disappearance of the $\{111\}$ facets is not the motion of the $\{111\}$ facet itself, but instead, it is the much faster evolution of the surrounding non-faceted interfaces, see Fig 6.

\subsection{Interface Shape Distributions}

ISDs of the microstructures are given in Fig. 8. They are read with the help of the shape index scale, $S \in[-1,1]$, in Fig. 1 . To compare multiple time steps, we scale the $C$ axis by the time-dependent length scale, $S_{v}$, and also fix the range of the color bar. In the as-cast sample, $t=0$ min., two concentrated peaks, i.e., $P(S, C) \geq 0.4$, are evident: the first is located at $S \approx 0.5$ and $C \approx 0.5 S_{v}$, and the second at $S \approx 0.5$ and $C \approx 2.5 S_{v}$. The latter peak represents the particle edges, while the former accounts for patches with near-planar shape. In other words, the as-cast microstructure has two characteristic length scales, at values that are 0.5 and 2.5 times $S_{v}$. Furthermore, the large tail in the distribution at $S=0.5$ indicates a dispersion in the sizes of the solid cylindrical-like shapes; the reason for this tail is that the interfacial patches must connect these two peak regions to form a continuous interface, and therefore must have a wide variety of "curvedness." As time increases, patches that are concave towards the solid $(S<0)$ almost completely disappear, and both the average shape index and average curvedness appear to increase, relative to $S_{v}$. For instance, at $t=50 \mathrm{~min}$., a peak is evident at $C \approx 3 S_{v}$ and $S \approx 0.75$, indicating that patches have cylindrical-like and spherical-like shape, consistent with the microstructure in Fig. 2(f). Since these ISDs are scaled by $S_{v}$, it is important to note that the variations of $S$ and $C$ are not due to the increased size-scale of the microstructure, but rather due to morphological changes alone.

We can consider shapes of patches independent of length scale, see Fig. 9. The histogram labels match those of Fig. 1. Since there is no length scale associated with

\footnotetext{
${ }^{4}$ The rate of this decay in fractional area, $A_{\{111\}} / S$, with time, $t$, warrants further study.
} 
these distributions, they should be invariant with time if the system is coarsening in a self-similar fashion. However, the probability of finding a patch with dome-like $(S \in$ $\left.\left[\frac{5}{8}, \frac{7}{8}\right)\right)$ or cap-like shape $\left(S \in\left[\frac{7}{8}, 1\right]\right)$ increases with coarsening time. Thus, even though the microstructures are evolving according to the $S_{v}^{-1} \propto t^{1 / 3}$ relationship, the structures are not self-similar. The lack of self-similarity is due to the loss of morphological anisotropy during the coarsening process.

\subsection{Texture Analysis}

Figs. 10(a-c) show three representative orientation maps of primary Si particles that have coarsened for two days at $600^{\circ} \mathrm{C}$. The mean crystallographic orientation of each grain of $\mathrm{Si}$ is colored according to the standard stereographic triangle on the bottom-right of Fig. 10(a), while non-indexed regions, belonging to the eutectic, are colored black. The grain boundaries were smoothed via 5 iterations of constrained Laplacian smoothing in MTEX software [55]. It can be seen that the Si particles consist of multiple grain boundaries that run along the long axes of the particles and that intersect the solid-liquid interface at the particle edges, suggesting the presence of coherent annealing twins $[56,57]$.

In order to quantify this observation, we measure the uncorrelated misorientation distribution function (MODF) using the discretized EBSD data taken from the interior region of a representative particle, as shown in Fig. 10(d). Since there may not be an orientation relationship between isolated $\mathrm{Si}$ particles in the microstructure, only single particles are considered for quantitative analysis. The MODF can be represented as angle and axis (inset) distributions in Fig. 10(e). The peak in the angle distribution near $0^{\circ}$ corresponds to low-angle, intra-granular misorientation noise, while the high probability of near $60^{\circ}$ angles corresponds to $<111>$ misorientation axes. Together, the $60^{\circ}<111>$ combination represents a $\Sigma 3 \mathrm{CSL}$, or a coherent twist grain boundary [45]. This angle-axis pair agrees with the IND results, since a $70.53^{\circ}$ symmetric tilt about $<110>$ is geometrically equivalent to the $60^{\circ}$ twist around $<111>$ : both configurations produce a $\Sigma 3$ CSL $[45,58]$. Consistent with the work of Ref. [58], our results demonstrate the high incidence of coherent twin boundaries in the polycrystalline Si particles.

\section{Discussion}

It is well known that the morphology of crystals results from an interplay of interfacial energy anisotropy and growth kinetics [59]. During the growth of primary Si particles, Moorthy and Howe [9] found that the faster, high-index orientations, tend to grow out and cease to exist, leaving the crystal shape bound by the slower growing $\{111\}$ planes [9]. This asymptotic shape is often referred to as the "kinetic Wulff shape" [59]. The kinetic Wulff shape can be different than the equilibrium Wulff shape. The former is determined by the low mobility crystal planes, while the latter is determined by the low energy planes [59]. For Si, the low energy and low mobility planes are of $\{111\}$-type. Qualitative observations from in situ microscropy suggest that the kinetic Wulff shape of Si in melt is octohedral in shape [12], while the equilibrium Wulff shape of $\mathrm{Si}$ is a tetrakaidecahedron ${ }^{56}$

\footnotetext{
${ }^{5}$ The equilibrium shape of $\mathrm{Si}$ was determined experimentally by Eaglesham et al. [11] by imaging in TEM small voids formed via He implantation in Si. All voids show the same broad features, i.e., large, flat $\{111\}$ facets, small $\{100\}$ facets, and no detectable $\{110\}$ or $\{311\}$ facets. Thus, to good approximation, the equilibrium shape of $\mathrm{Si}$ is a tetrakaidecahedron, or a polyhedron with 14 faces [11].

${ }^{6}$ Barinovs et al. [60] have demonstrated via molecular dynamics simulation with Stillinger-Weber potential that the calculated equilibrium shape of $\mathrm{Si}$ crystal in the melt is geometrically similar to the equilibrium shape of the Si solid-vapor interface, observed experimentally by Eaglesham et al. [11].
} 
dominated by large $\{111\}$ facets and smaller $\{100\}$ surfaces [11]. Furthermore, since the eutectic temperature in the $\mathrm{Al}-\mathrm{Si}$ system $\left(577^{\circ} \mathrm{C}\right)$ is well below the roughening transition temperature for $\mathrm{Si}\left(757^{\circ} \mathrm{C}[61]\right)$, at which steps are spontaneously created on $\{111\}$, it is expected that the equilibrium Wulff shape is fully faceted for temperatures considered in this study.

During the coarsening of Si particles, we found that $S_{v}^{-1}$ varies as $t^{1 / 3}$, see Fig. 3, suggesting that long-range diffusion of solute is an important factor during the coarsening process. However, unlike classical coarsening theories [34, 35], the microstructural evolution in this case is not governed by the interfacial free energy of the $\{111\}$ planes. If the driving force is the reduction in total interfacial energy, then the particle structure would approach the equilibrium Wulff shape, and correspondingly, the fractional area of $\{111\}$ planes would increase with time. However, the opposite trend is reported here, see Fig. 7. In general, the particle structure becomes less faceted and more isotropic with time, as seen in the INDs and ISDs of Figs. 4 and 5, and Figs. 8 and 9, respectively.

The Si particles of this study have "mixed interfaces", i.e., partially faceted and partially rounded. From the 3D reconstructions in Figs. 2 and 6, we see that the $\{111\}$ facets are slow-moving during the microstructural evolution. Since the interfaces are evolving during coarsening, the driving forces at $\{111\}$ planes are small compared to those encountered during rapid crystal growth, and thus the kinetic limitations of step formation can be particularly difficult to overcome without the benefit of defects [26]. This is consistent with the Jackson $\alpha$ factor for the Si $\{111\}$ interface [16].

On the other hand, the particle edges appear non-faceted and thus have the kink sites necessary for interfacial propagation. The exposure of these less close-packed planes of Si to the liquid results in rapid lateral growth and a decrease in the area of the broad equilibrium $\{111\}$ facets. If the explanation put forth by Moorthy and Howe [9] is appropriate, then the partially rounded interfaces that we see during the growth of Si particles may in fact be comprised of low mobility, sub-micrometer scale $\{111\}$ facets. If such micro-facets exist, however, they are well below the spatial resolution of these X-ray tomography experiments. Another explanation is that these more rapidly moving interfaces could be either (i) atomically rough, or (ii) highly defective, with a high incidence of dislocations or re-entrant twin grooves at the edges of the faceted crystals. However, the particle edges are neither thermodynamically rough [62], since the annealing temperature is well below that of the roughening transition, nor are they kinetically rough [63, 64], due to relatively slow interfacial velocities during growth. Instead, EBSD measurements provide evidence for twin defects, which may contribute significantly to the growth process.

In particular, we find that $\Sigma 3$ twin boundaries intersect the solid-liquid interface, see Fig. 10. These coherent annealing twins may have formed during the solidification of the primary silicon particles from the melt. The twins are formed and terminated by errors in the stacking of $\{111\}$ planes during grain growth $[65,66,67]$. It is also conceivable that the coherent twins arise naturally during the coarsening process. For instance, the particle thickness (in the direction perpendicular to the long axis) increased by roughly $20 \%$ or $10 \mu \mathrm{m}$ in $10 \mathrm{~min}$., see Fig. 7 . In this time interval, growth accidents on the $\{111\}$ facets may have promoted the formation of coherent twin boundaries, whose spacing may be on the order of a few micrometers, as shown in Fig. 10.

Twins are well known to act as a source of steps. For example, the presence of at least two such twin planes can give rise to the twin plane re-entrant edge (TPRE) mechanism: growth on the re-entrant corners generates more re-entrant corners, thereby providing unlimited steps for interfacial attachment and detachment $[18,19,68,69]$. Thus, the rate of growth at the multiply twinned particle edges is limited by the rate at which solute 
atoms diffuse to the surface. This implies that the growth of the particle is determined principally by growth at its edges; hence why the average length scale of the system, $S_{v}^{-1}$, varies as $t^{1 / 3}$. In the limit of very long coarsening times, it is anticipated that the particle edges would completely overgrow the relatively immobile facets, leading to a completely isotropic structure. In other words, the morphology of the defective crystals could indeed be quite isotropic and thus distinct from the equilibrium Wulff shape of Si, a tetrakaidecahedron dominated by large $\{111\}$ facets and smaller $\{100\}$ surfaces [11].

A potentially confounding process that needs to be considered is the effect of quenching on the microstructural evolution. During quenching, we expect that the $\{111\}$ facets of Si fail to respond to the rapid fall in temperature, due to their low interfacial mobility. However, the effects of quenching on the particle edges are unknown. To address this issue, we very recently observed in situ similar rounding of primary Si particlees in both $\mathrm{Al}-29.9 \mathrm{wt} \% \mathrm{Si}$ and $\mathrm{Al}-32 \mathrm{wt} \% \mathrm{Si}-15 \mathrm{wt} \% \mathrm{Cu}$ alloys [10], when these samples were held isothermally in the two-phase solid-liquid regime. This suggests that thermal cycling has negligible influence on the morphological changes observed in this work.

\section{Conclusions}

Four-dimensional phase contrast X-ray tomography experiments were performed on Al-29.9wt\%Si alloy during coarsening. While the morphology of the as-cast sample is complex and faceted, the morphologies at later times are less faceted and more isotropic. The structures are not evolving in a self-similar manner, as quantitatively confirmed by the interface shape distributions, yet we still observe that a characteristic length, the inverse of the surface area per unit volume, still scales as $t^{1 / 3}$. More specifically, the interface normal distributions show that the Si particles are not evolving towards their fully-faceted equilibrium Wulff shape. This is because the particle edges move rapidly due to the prevalence of defects, and eventually overgrow the relatively immobile $\{111\}$ faces. Thus, the evolution of the particle structure is determined principally by growth and dissolution at its edges and its defect structure.

\section{Acknowledgements}

The analysis of the data was supported by the Multidisciplinary University Research Initiative (MURI) under award AFOSR FA9550-12-1-0458. Additional support was provided for AJS by the NSF Graduate Research Fellowship under grant no. DGE-1324585, and JWG by the DOE NNSA Stewardship Science Graduate Fellowship under grant no. DE-FC52-08NA28752. The sample preparation and data acquisition were supported by the DOE under contract no. DE-FG02-99ER45782. We thank the staff at the TOMCAT beamline, especially Dr. Sarah Irvine and Gordan Mikuljan; Dr. Carla Shute, Northwestern University, for polishing, etching, and microscopy assistance; Ashley Paz y Puente, Northwestern University, for assistance with the ashing furnace; and Newlin Weatherford, Northwestern University, for assistance with stereological analysis. JLF also acknowledges the CCMX and PSI for funding.

This work made use of the EPIC facility (NUANCE Center-Northwestern University), which has received support from the MRSEC program (NSF DMR-1121262) at the Materials Research Center; the Nanoscale Science and Engineering Center (NSF EEC-0647560) at the International Institute for Nanotechnology; and the State of Illinois, through the International Institute for Nanotechnology. 


\section{Appendix A. Analysis of Misorientation Angles Using Tomographic Data}

Prior to spherical k-means (SKM) clustering, the interface normals are segmented on the hypersphere with an appropriate threshold; we use the threshold $4 \times 10^{-4}$. The results of SKM clustering [43] of the interface normals from the as-cast sample (Fig. 4) are shown in Fig. 11. To reiterate, SKM clustering is performed on the unit hypersphere, but is shown here as a Mercator projection for the sake of clarity. The different colors represent the different clusters, each of which has a centroid vector $\{h k l\}$ shown as a black point. The 17 cluster centroids identified are labelled $A$ through $Q$ for further analysis. Each cluster is assumed to correspond to only one face on a particle.

In the cubic system, two $\{111\}$ facets that meet at a large angle twin boundary [44] around $<110\rangle$ have a misorientation angle of either $70.53^{\circ}, 109.47^{\circ}$, or $180^{\circ}$; the latter angle accounts for inversion symmetry. Thus, we compute the angles between all 17 centroid vectors and compare to these three misorientation angles, see Table A.1. The angle, $\alpha_{i j}$, between centroid vectors $i$ and $j$, both of unit length, is given by

$$
\alpha_{i j}=\cos ^{-1}\left(h_{i} h_{j}+k_{i} k_{j}+l_{i} l_{j}\right)
$$

As an intermediate step, if $\alpha_{i j}$ matches any of $70.53^{\circ}, 109.77^{\circ}$, or $180^{\circ}$ within an error of $10 \%$, cell $i j$ in Table A.1 is highlighted in red. Note, however, that the angle between two unrelated facets (that do not meet at a twin boundary) may, by chance, match any one of these three critical angles. Therefore, it is imperative to accept or reject the red assignments by inspecting the real-space reconstructions of these facet pairs. "Rejections" of two unrelated facets $i$ and $j$ are denoted in Table A.1 by a diagonal line through $\alpha_{i j}$.

Using this procedure, we identify the poles $A, B, C, D, G, H, I, L, M, N, O$, and $Q$ as $\{111\}$ facets, or 12 of the 17 original centroid vectors. There may be, in fact, more $\{111\}$ facets in the ROI, e.g., those that meet at small angle twin boundaries via displacement twinning [44], but these facets are computationally inaccessible with this approach.

\section{References}

[1] M. G. Day, A. Hellawell, The microstructure and crystallography of aluminiumsilicon eutectic alloys, Proc. R. Soc. A 305 (1968) 473-491.

[2] M. M. Haque, M. A. Maleque, Effect of process variables on structure and properties of aluminium-silicon piston alloy, J. Mater. Process Technol. 77 (1998) 122-128.

[3] V. S. Zolotorevsky, N. A. Belov, M. V. Glazoff, Casting Aluminum Alloys, Elsevier, 2010.

[4] L. Ratke, P. W. Voorhees, Growth and Coarsening: Ostwald Ripening in Material Processing, Springer, 2002.

[5] H. Ye, An overview of the development of al-si alloy based material for engine applications, J. Mater. Eng. Perform. 12 (2003) 288-297.

[6] G. Heiberg, K. Nogita, A. K. Dahle, L. Amberg, Columnar to equiaxed transition of eutectic in hypoeutectic aluminium-silicon alloys, Acta Mater. 50 (2002) 2537-2546.

[7] L. Zhang, D. G. Eskin, A. Mirouz, L. Katgerman, Formation of microstructure in al-si alloys under ultrasonic melt treatment, Light Met. (2012) 999-1004. 
[8] F. Czerwinski, The basics of modern semi-solid metal processing, JOM 58 (2006) $17-20$.

[9] S. K. E. Moorthy, J. M. Howe, Kinetic analyses of the growth and dissolution phenomena of primary si and $\alpha$-al in partially molten al-si (-cu-mg) alloy particles using in situ transmission electron microscopy, Metall. Mater. Trans. A 42 (2011) 16671674.

[10] A. J. Shahani, X. Xiao, P. W. Voorhees, In-situ synchrotron tomographic investigation of the coarsening evolution of al-si-cu alloy, Mater. Sci. Eng. AManuscript in Preparation.

[11] D. J. Eaglesham, A. E. White, L. C. Feldman, N. Moriya, D. C. Jacobson, Equilibrium shape of si, Phys. Rev. Lett. 70 (1993) 1643-1647.

[12] X. Yang, K. Fujiwara, K. Maeda, J. Nozawa, H. Koizumi, S. Uda, Crystal growth and equilibrium crystal shapes of silicon in the melt, Prog. Photovolt: Res. Appl. 20 .

[13] P. A. Apte, X. C. Zeng, Anisotropy of crystal-melt interfacial free energy of silicon by simulation, Appl. Phys. Lett. 92 (2008) 221903.

[14] G. H. Gilmer, A. F. Bakker, Molecular dynamics simulations of steps at crystal surfaces, MRS Proc. 209.

[15] K. A. Jackson, Crystal growth kinetics, Mater. Sci. Eng. 65 (1984) 7-13.

[16] K. M. Beatty, K. A. Jackson, Monte carlo modeling of silicon crystal growth, J. Cryst. Growth 211 (2011) 13-17.

[17] W. Obreten, D. Kashchiev, V. Bostanov, Unified description of the rate of nucleation -mediated crystal growth, J. Cryst. Growth 96 (1989) 843-848.

[18] K. Fujiwara, K. Maeda, N. Usami, K. Nakajima, Growth mechanism of si-faceted dendrites, Phys. Rev. Lett. 101 (2008) 0555031-0555034.

[19] K. Fujiwara, K. Maeda, N. Usami, G. Sazaki, Y. Nose, K. Nakajima, Formation mechanism of parallel twins related to si-facetted dendrite growth, Scripta Mater. 57 (2007) 81-84.

[20] A. D. Gamalski, P. W. Voorhees, C. Ducati, R. Sharma, S. Hoffmann, Twin plane re-entrant mechanism for catalytic nanowire growth, Nano Lett. 14 (2014) 12881292.

[21] R. E. Napolitano, H. Meco, C. Jung, Faceted solidification morphologies in lowgrowth-rate al-si eutectics, JOM 56 (2004) 16-21.

[22] J. L. Fife, Three-dimensional characterization and real-time interface dynamics of aluminum-copper dendritic microstructures, Ph.D. thesis, Northwestern University (June 2009).

[23] R. Mendoza, Morphological and topological characterization of coarsened dendritic microstructures, Ph.D. thesis, Northwestern University (june 2004). 
[24] D. J. Rowenhorst, P. W. Voorhees, Measurement of interfacial evolution in three dimensions, Ann. Rev. Mater. Res. 42 (2012) 105-124.

[25] J. L. Fife, J. W. Gibbs, E. B. Gulsoy, C.-L. Park, K. Thornton, P. W. Voorhees, The dynamics of interfaces during coarsening in solid-liquid systems, Acta Mater. 70 (2014) 66-78.

[26] E. B. Gulsoy, A. J. Shahani, J. W. Gibbs, J. L. Fife, P. W. Voorhees, Fourdimensional morphological evolution of coarsening of aluminum-silicon alloy using phase contrast x-ray tomography, Mater. Trans., JIM 55 (2014) 161-164.

[27] A. J. Shahani, E. B. Gulsoy, J. W. Gibbs, J. L. Fife, P. W.Voorhees, An integrated approach to the processing of four-dimensional datasets from phase contrast x-ray tomography, Opt. Express 22 (2014) 24606-24621.

[28] Materials Preparation Center, Ames Laboratory, US DOE Basic Energy Sciences, Ames, IA, USA, www.mpc.ameslab.gov.

[29] M. Stampanoni, A. Groso, A. Isenegger, G. Mikuljan, Q. Chen, A. Bertrand, S. Henein, R. Betemps, U. Frommherz, P. Bhler, D. Meister, M. Lange, R. Abela, Trends in synchrotron-based tomographic imaging: the sls experience, Proc. of SPIE (2006) 63180M-63180M14.

[30] D. Paganin, S. C. Mayo, T. E. Gureyev, P. R. Miller, S. W. Wilkins, Simultaneous phase and amplitude extraction from a single defocused image of a homogeneous object, J. Microsc. 206 (2002) 33-40.

[31] F. Marone, M. Stampanoni, Regridding reconstruction algorithm for real-time tomographic imaging, J. Synchrotron Rad. (2012) 1029-1037.

[32] G. Lovric, S. Barré, J. C. Schittny, M. Roth-Kleiner, M. Stampanoni, R. Mokso, Dose optimization approach to fast x-ray microtomography of the lung alveoli, J. Appl. Cryst. 46 (2013) 856-860.

[33] M. Botsch, L. Kobbelt, M. Pauly, P. Alliez, B. Levy, Polygon Mesh Processing, A.K. Peters, 2010.

[34] I. M. Lifshitz, V. V. Slyozov, The kinetics of precipitation from supersaturated solid solutions, J. Phys. Chem. Solids 19 (1961) 35-50.

[35] C. Wagner, Theorie der alterung von niederschlägen durch umlösen (ostwaldreifung), Z. Elektrochem 65 (1961) 581-591.

[36] S. P. Marsh, M. E. Glicksman, Overview of geometric effects on coarsening of mushy zones, Metall. Mater. Trans. A 27A (1996) 557-567.

[37] A. Baddeley, E. B. V. Jensen, Stereology for Statisticians, Chapman and Hall, 2005.

[38] E. E. Underwood, Quantitative Stereology, Addison-Wesley, 1970.

[39] D. Kammer, P. W. Voorhees, The morphological evolution of dendritic microstructures during coarsening, Acta Mater. 54 (2006) 1549-1558. 
[40] J. L. Fife, P. W. Voorhees, The morphological evolution of equiaxed dendritic microstructures during coarsening, Acta Mater. 57 (2009) 2418-2428.

[41] B. D. Cullity, S. R. Stock, Elements of X-ray Diffraction, Addison-Wesley, 1956.

[42] C. F. Marcus, The stereographic projection in vector notation, Math. Assoc. Am 39 (1966) 100-102.

[43] I. S. Dhillon, D. S. Modha, Concept decompositions for large sparse text data using clustering, Mach. Learn (2001) 143-175.

[44] M. Shamsuzzoha, L. M. Hogan, Crystal morphology of unmodified aluminum-silicon eutectic microstructures, J. Cryst. Growth 76 (1986) 429-439.

[45] H. Mykura, A checklist of cubic coincidence site lattice relations, in: R. W. Baluffi (Ed.), Grain Boundary Structure and Kinetics, ASM International, 1980.

[46] J. E. Taylor, Overview no. 98. ii: Mean curvature and weighted mean curvature, Acta Metall. 40 (1992) 1475-1485.

[47] J. W. Cahn, D. W. Hoffman, A vector thermodynamics for anisotropic surfaces: Ii. curved and faceted surfaces, Acta Metall. 22 (1974) 1205-1214.

[48] D. W. Hoffman, J. W. Cahn, A vector thermodynamics for anisotropic surfaces: I. fundamentals and applications to plane surface junctions, Surf. Sci. 31 (1972) 368388.

[49] W. C. Carter, A. Roosen, J. W. Cahn, J. E. Taylor, Shape evolution by surface diffusion and surface attachment limited kinetics on completely faceted surfaces, Acta Metall. 43 (1995) 4309-4323.

[50] A. Roosen, J. E. Taylor, Modeling crystal growth in a diffusion field using fullyfaceted interfaces, J. Comp. Phys. 114 (1994) 113-128.

[51] W. Kühnel, Differential Geometry: Curves, Surfaces, and Manifolds, Am. Math. Soc., 2003.

[52] J. L. Fife, P. W. Voorhees, Self-similar microstructural evolution of dendritic solidliquid mixtures during coarsening, Scripta Mater. 60 (2009) 839-842.

[53] D. J. Rowenhorst, private communication (2014).

[54] J. Koenderink, A. van Doorn, Surface shape and curvature scales, Image Vision Comput. 10 (1992) 557-565.

[55] F. Bachmann, R. Hielscher, H. Schaeben, Texture analysis with MTEX: Free and open source software toolbox, Sol. St. Phen. 160 (2010) 63-68.

[56] V. Randle, O. Engler, Texture Analysis: Macrotexture, Microtexture, and Orientation Mapping, Gordon and Breach, 2000.

[57] S. Mahajan, C. S. Pande, M. A. Imam, B. B. Rath, Formation of annealing twins in f.c.c. crystals, Acta Mater. 45 (1997) 2633-2638. 
[58] S. Ratanaphan, Y. Yoon, G. S. Rohrer, The five-parameter grain boundary distribution of polycrystalline silicon, J. Mater Sci. 49 (2014) 4938-4945.

[59] R. F. Sekerka, Theory of crystal growth morphology, in: G. Müller, J. J. Métois, P. Rudolph (Eds.), Crystal Growth: from Fundamentals to Technology, Elsevier, 2004.

[60] G. Barinovs, A. Sabanskis, A. Muiznieks, Study of silicon crystal formation based on molecular dynamics simulation results, J. Cryst. Growth 391 (2014) 13-17.

[61] A. K. Tan, C. K. Ong, H. S. Tan, Monte carlo simulation of crystal growth from silicon melt, Semicond. Sci. Technol. 3 (1988) 1-5.

[62] W. K. Burton, N. Cabrera, F. C. Frank, The growth of crystals and the equilibrium structure of their surfaces, Philos. Trans. A243 (1951) 299-358.

[63] O. E. Temkin, Phenomenological kinetics of the motion of a phase boundary, Sov. Phys. Cryst. 15 (1971) 767-772.

[64] O. E. Temkin, Kinetic phase transition during a phase conversion in a binary alloy, Sov. Phys. Cryst. 15 (1971) 773-780.

[65] H. C. H. Carpenter, S. Tamura, The formation of twinned metallic crystals, Proc. R. Soc. 113 (1926) 161-182.

[66] E. Burke, The formation of annealing twins, Trans. Am. Inst. Min. Engrs. 188 (1950) 1324-1328.

[67] H. Gleiter, The formation of annealing twins, Acta Metall. 17 (1969) 1421-1428.

[68] D. R. Hamilton, R. G. Siedensticker, Propagation mechanism of germanium dendrites, J. Appl. Phys. 31 (1960) 1165-1168.

[69] R. S. Wagner, On the growth of germanium dendrites, Acta Metall. 8 (1960) 57-60. 


\section{List of Figures}

$1 \quad$ Illustration of shape $(S)$ index scale, divided into nine categories: spherical cup for $S \in\left[-1,-\frac{7}{8}\right)$, trough for $S \in\left[-\frac{7}{8},-\frac{5}{8}\right)$, rut for $S \in\left[-\frac{5}{8},-\frac{3}{8}\right)$, saddle rut for $S \in\left[-\frac{3}{8},-\frac{1}{8}\right)$, saddle for $S \in\left[-\frac{1}{8}, \frac{1}{8}\right)$, saddle ridge for $S \in\left[\frac{1}{8},-\frac{3}{8}\right)$, ridge for $S \in\left[\frac{3}{8}, \frac{5}{8}\right)$, dome for $S \in\left[\frac{5}{8}, \frac{7}{8}\right)$, and spherical cap for $S \in\left[\frac{7}{8}, 1\right]$. Shown shapes are of identical curvedness, $C=1$. Adapted from Koenderink et al. [54]. . . . . . . . . . . . . . . . . . . .

23 D microstructure coarsening in time. Light gray regions are the primary Si particles while the dark gray background is the eutectic. The as-cast sample in (a) was coarsened in furnace at $590^{\circ} \mathrm{C}$ for (b) 10 min., (c) 20 min., (d) 30 min., (e) $40 \mathrm{~min}$., and (f) $50 \mathrm{~min}$. A ROI of $296 \times 296 \times 159 \mu \mathrm{m}^{3}$ is shown $[27] \ldots \ldots \ldots \ldots \ldots \ldots$

3 (a) Log-log plot of inverse surface area per unit volume at different coarsening times for Al-29.9wt\% Si. Blue data points are measured from XRT, and the orange data points are measured from optical metallography. In the tomography data, error bars are estimated from the number of mis-classified voxels, in comparison to a few hand-segmented images [27], while in the metallography data, error bars are determined from counting statistics during stereological analysis. (b-e) Optical micrographs collected at the same magnification (92x) during the coarsening evolution of silicon particles (black) in a eutectic matrix (white). . . . . . . . . . . . .

4 Interface normal distribution (IND) for the as-cast sample, $t=0$ min., depicted as a Mercator projection of the unit hypersphere. The IND contains several poles indicating a highly faceted structure. Those poles that are boxed (A-D) belong to the same connected component and to the $\{111\}$ family, and are used in subsequent analysis. . . . . . . . . . . . . .

5 Evolution of $\{111\}$ Mercator poles (A-D) with coarsening time. Fixing the range of the color bar and the range of values $\left(\theta^{\prime}, \phi^{\prime}\right)$ that are indicative of each of the four facets allows multiple coarsening times to be compared. In general, pole intensities decrease with time for facets A-D. . . . . . . .

6 Specific Microstructural Location (SML) figures, depicting $\{111\}$ patches (A-D) in red, for the (a) as-cast sample, and after (b) 10 min., (c) 20 min., (d) 30 min., (e) $40 \mathrm{~min}$., and (f) $50 \mathrm{~min}$. of coarsening at $590^{\circ} \mathrm{C}$. The mesh patches belonging to $\{111\}$ poles A-D were determined by segmenting those poles on the unit hypersphere with a threshold of $4 \times 10^{-4}$. The misorientation between the two particles is $70.5^{\circ}<110>$, as indicated in (a). The misorientation axis points out of the page. . . . . . . . . .

7 Area of $\{111\}$ patches in connected component $A B C D, A_{\{111\}}$, over total surface area, $S$, versus coarsening time. Fractional areas, $A_{\{111\}} / S$, were determined by summing over bins in the IND which had values above the threshold of $4 \times 10^{-4}$. Errors bars are due to binned patches that do not belong to connected component $A B C D$, but rather to similarly-oriented particles (roughly 5-8\% error). The trend indicates that the Si particles evolve away from their equilibrium Wulff shape. . . . . . . . . . . . 
8 Interface shape distributions (ISDs) using the alternative $S$ versus $C$ classification [54], for the same microstructures shown in Fig. 2: (a) $t=0$ min. and $S_{v}^{-1}=19 \mu \mathrm{m}$, (b) $t=10 \mathrm{~min}$. and $S_{v}^{-1}=31 \mu \mathrm{m}$, (c) $t=20$ min. and $S_{v}^{-1}=42 \mu \mathrm{m}$, and (d) $t=50 \mathrm{~min}$. and $S_{v}^{-1}=53 \mu \mathrm{m}$. Scaling the curvedness, $C$, by $S_{v}$ and fixing the range of the color bar allow multiple coarsening times to be compared. Dashed lines at $S=\{-0.5,0,0.5\}$ serve as markers for liquid cylindrical-like shapes ("ruts"), saddle-like shapes, and solid cylindrical-like shapes ("ridges"), respectively. . . . . . . . . .

9 The probability of finding a patch of a given shape as a function of time, where (a) is 0 min., (b) is $10 \mathrm{~min}$. , (c) is $20 \mathrm{~min}$. , and (d) is $50 \mathrm{~min}$., of elapsed coarsening time. These plots are for the same microstructures measured in Fig. 8. We use the shape index scale of Fig. 1. The fractions of domes and spherical caps increase with time, suggesting that the coarsening evolution of primary $\mathrm{Si}$ particles is not self-similar. . . . . . .

10 (a-c) Orientation maps of three representative Si particles that have coarsened for two days at $600^{\circ} \mathrm{C}$. The mean crystallographic orientation of each grain of $\mathrm{Si}$ is colored according to the standard stereographic triangle on the bottom-right of (a), while non-indexed regions belonging to the eutectic are colored black. (d) Discretized EBSD data of boxed region, where intra-granular variations and grain boundaries are apparent, and from which the uncorrelated misorientation distribution function (MODF) is calculated. The resulting MODF can then be represented as (e) misorientation angle and axis (inset) distributions. . . . . . . . . . . . .

11 Results of spherical k-means (SKM) clustering of the interface normals from the as-cast microstructure. Different colors indicate different clusters; black points are the locations of the centroid vectors, $\{h k l\}$. We identify a total of 17 clusters, labelled $A$ through $Q \ldots \ldots$. . . . . . 


\section{FIGURES}

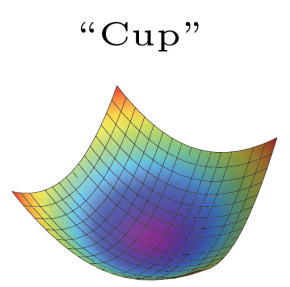

Solid

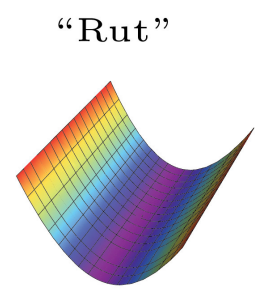

Solid

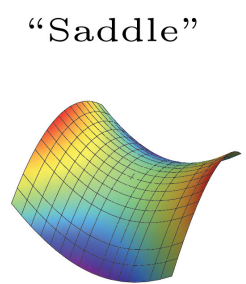

Solid

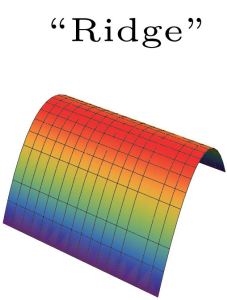

Solid

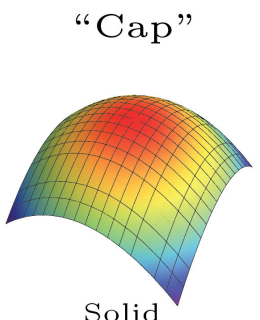

Solid

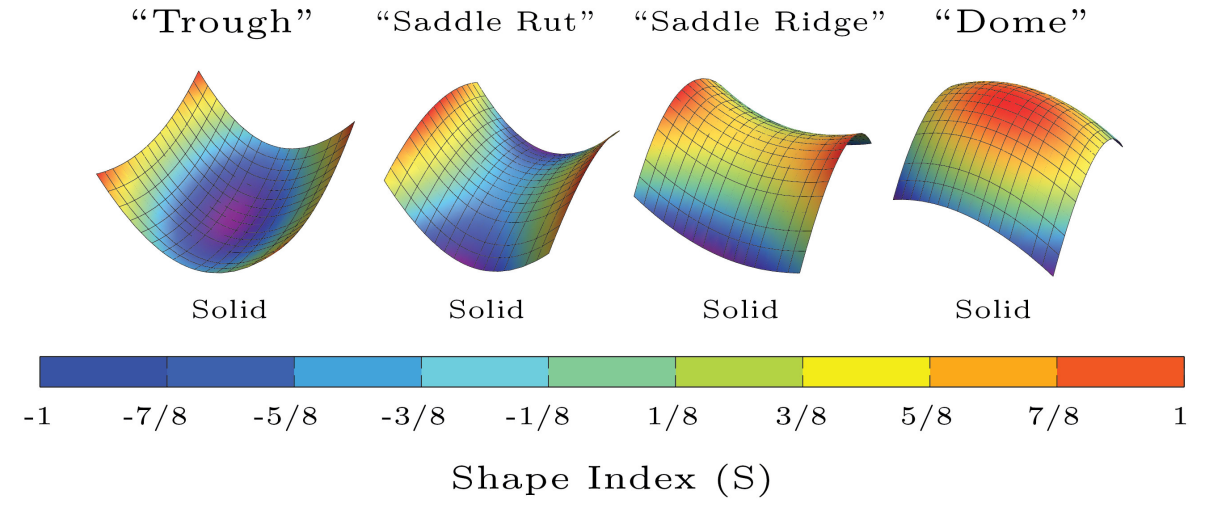

Figure 1: Illustration of shape $(S)$ index scale, divided into nine categories: spherical cup for $S \in$ $\left[-1,-\frac{7}{8}\right)$, trough for $S \in\left[-\frac{7}{8},-\frac{5}{8}\right)$, rut for $S \in\left[-\frac{5}{8},-\frac{3}{8}\right)$, saddle rut for $S \in\left[-\frac{3}{8},-\frac{1}{8}\right)$, saddle for $S \in\left[-\frac{1}{8}, \frac{1}{8}\right)$, saddle ridge for $S \in\left[\frac{1}{8},-\frac{3}{8}\right)$, ridge for $S \in\left[\frac{3}{8}, \frac{5}{8}\right)$, dome for $S \in\left[\frac{5}{8}, \frac{7}{8}\right)$, and spherical cap for $S \in\left[\frac{7}{8}, 1\right]$. Shown shapes are of identical curvedness, $C=1$. Adapted from Koenderink et al. [54]. 


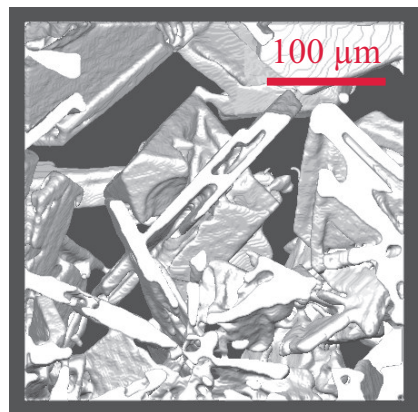

(a)

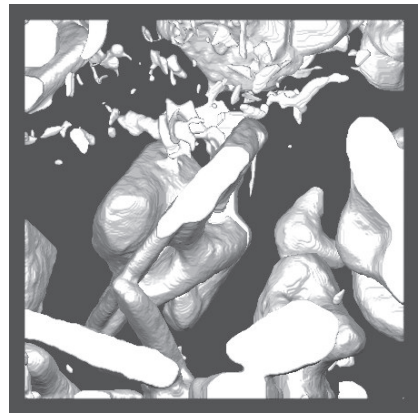

(d)

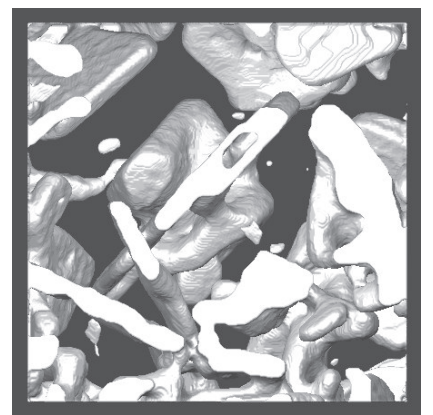

(b)

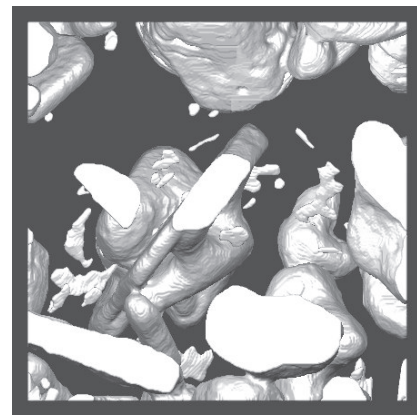

(e)

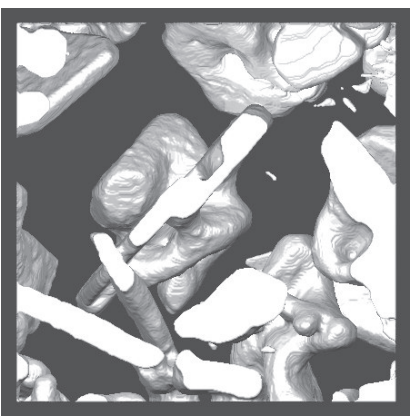

(c)

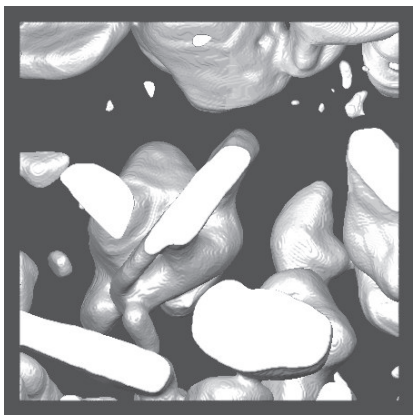

(f)

Figure 2: 3D microstructure coarsening in time. Light gray regions are the primary Si particles while the dark gray background is the eutectic. The as-cast sample in (a) was coarsened in furnace at $590^{\circ} \mathrm{C}$ for (b) $10 \mathrm{~min}$., (c) $20 \mathrm{~min}$., (d) $30 \mathrm{~min}$., (e) $40 \mathrm{~min}$., and (f) $50 \mathrm{~min}$. A ROI of $296 \times 296 \times 159 \mu \mathrm{m}^{3}$ is shown $[27]$. 

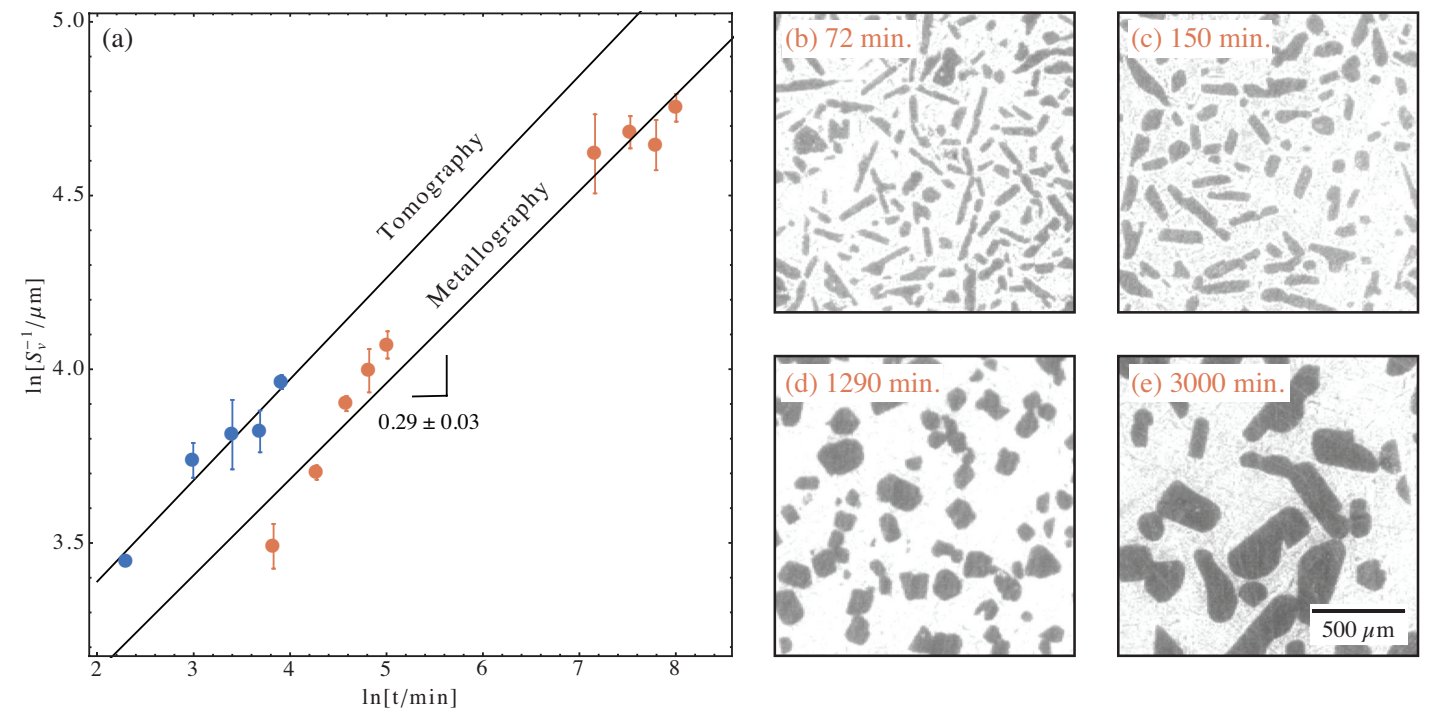

Figure 3: (a) Log-log plot of inverse surface area per unit volume at different coarsening times for Al-29.9wt\%Si. Blue data points are measured from XRT, and the orange data points are measured from optical metallography. In the tomography data, error bars are estimated from the number of mis-classified voxels, in comparison to a few hand-segmented images [27], while in the metallography data, error bars are determined from counting statistics during stereological analysis. (b-e) Optical micrographs collected at the same magnification $(92 \mathrm{x})$ during the coarsening evolution of silicon particles (black) in a eutectic matrix (white). 


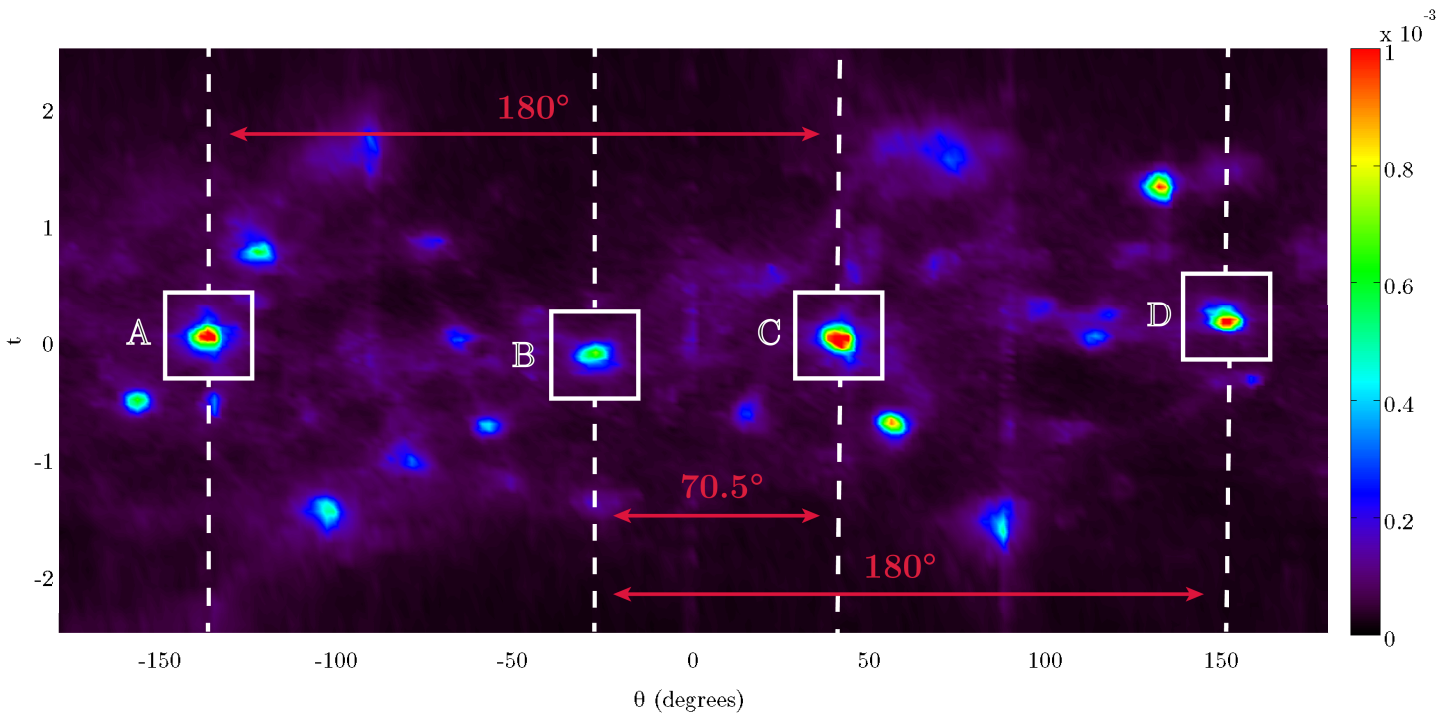

Figure 4: Interface normal distribution (IND) for the as-cast sample, $t=0$ min., depicted as a Mercator projection of the unit hypersphere. The IND contains several poles indicating a highly faceted structure. Those poles that are boxed (A-D) belong to the same connected component and to the $\{111\}$ family, and are used in subsequent analysis. 


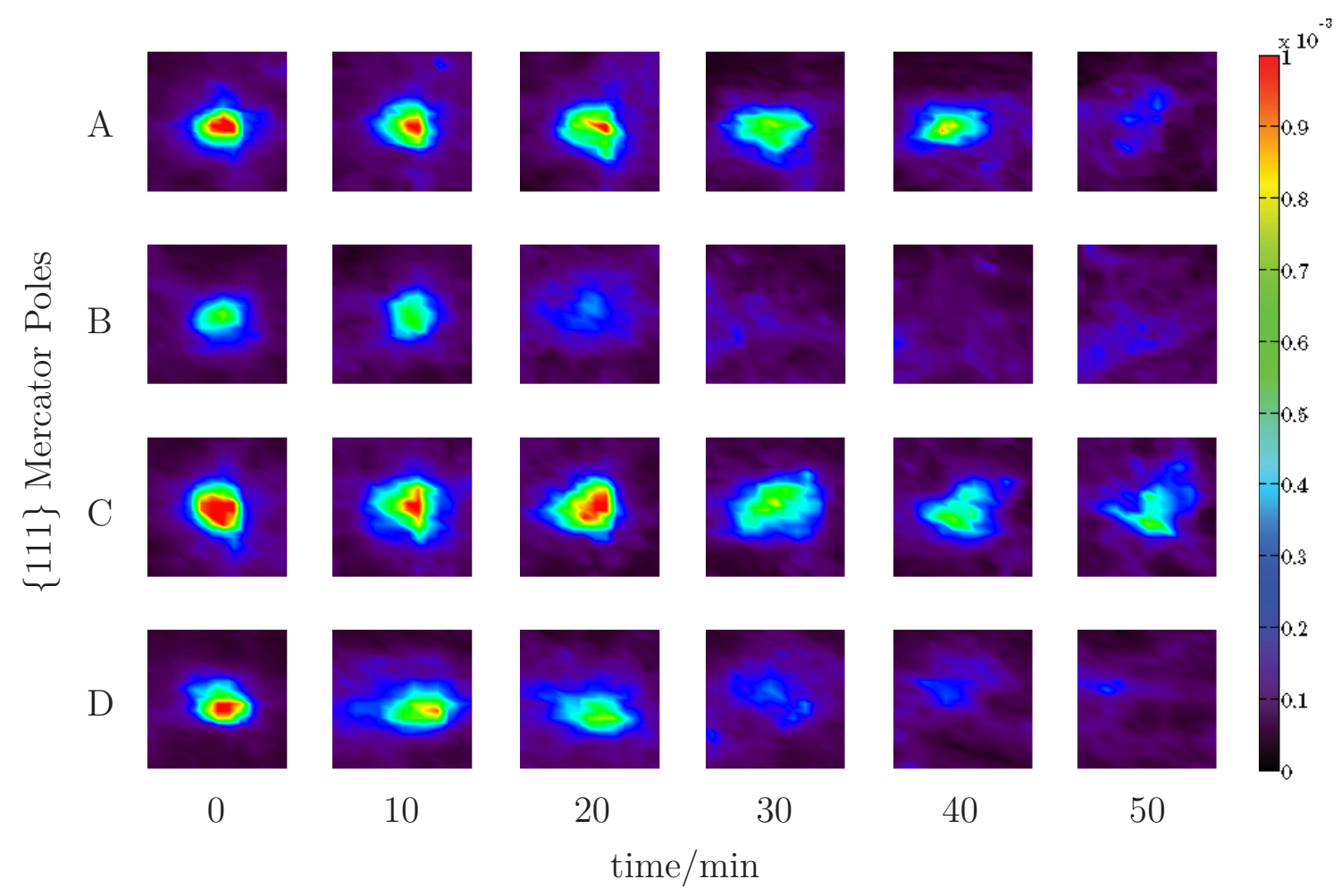

Figure 5: Evolution of $\{111\}$ Mercator poles (A-D) with coarsening time. Fixing the range of the color bar and the range of values $\left(\theta^{\prime}, \phi^{\prime}\right)$ that are indicative of each of the four facets allows multiple coarsening times to be compared. In general, pole intensities decrease with time for facets A-D. 


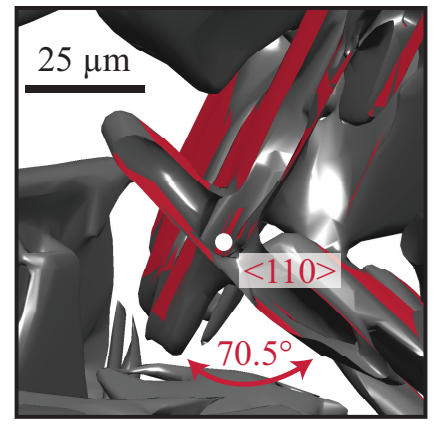

(a)

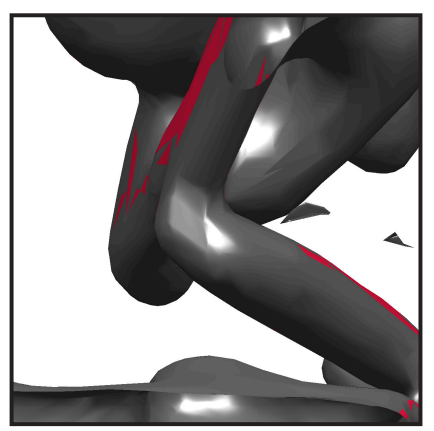

(d)

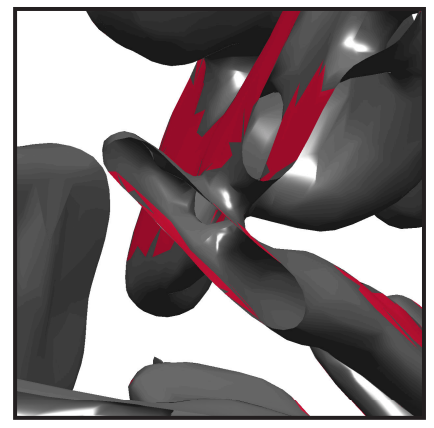

(b)

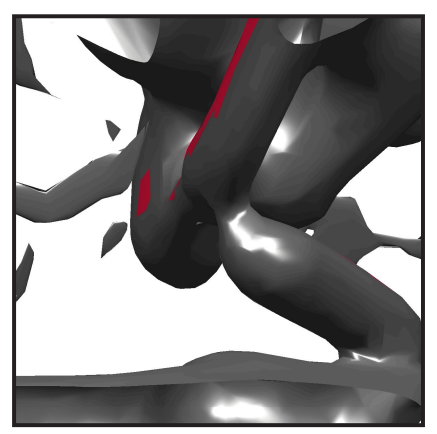

(e)

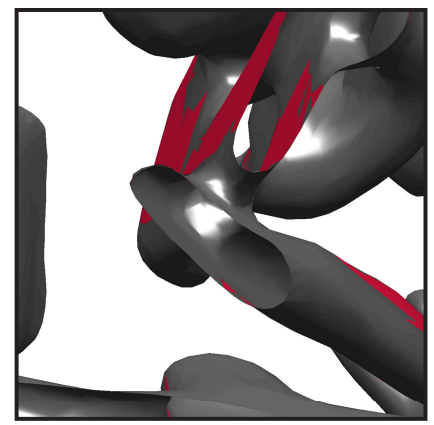

(c)

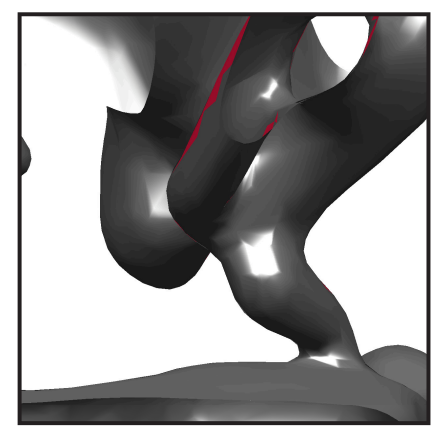

(f)

Figure 6: Specific Microstructural Location (SML) figures, depicting $\{111\}$ patches (A-D) in red, for the (a) as-cast sample, and after (b) $10 \mathrm{~min}$., (c) $20 \mathrm{~min}$., (d) $30 \mathrm{~min}$., (e) $40 \mathrm{~min}$., and (f) $50 \mathrm{~min}$. of coarsening at $590^{\circ} \mathrm{C}$. The mesh patches belonging to $\{111\}$ poles A-D were determined by segmenting those poles on the unit hypersphere with a threshold of $4 \times 10^{-4}$. The misorientation between the two particles is $70.5^{\circ}<110>$, as indicated in (a). The misorientation axis points out of the page. 


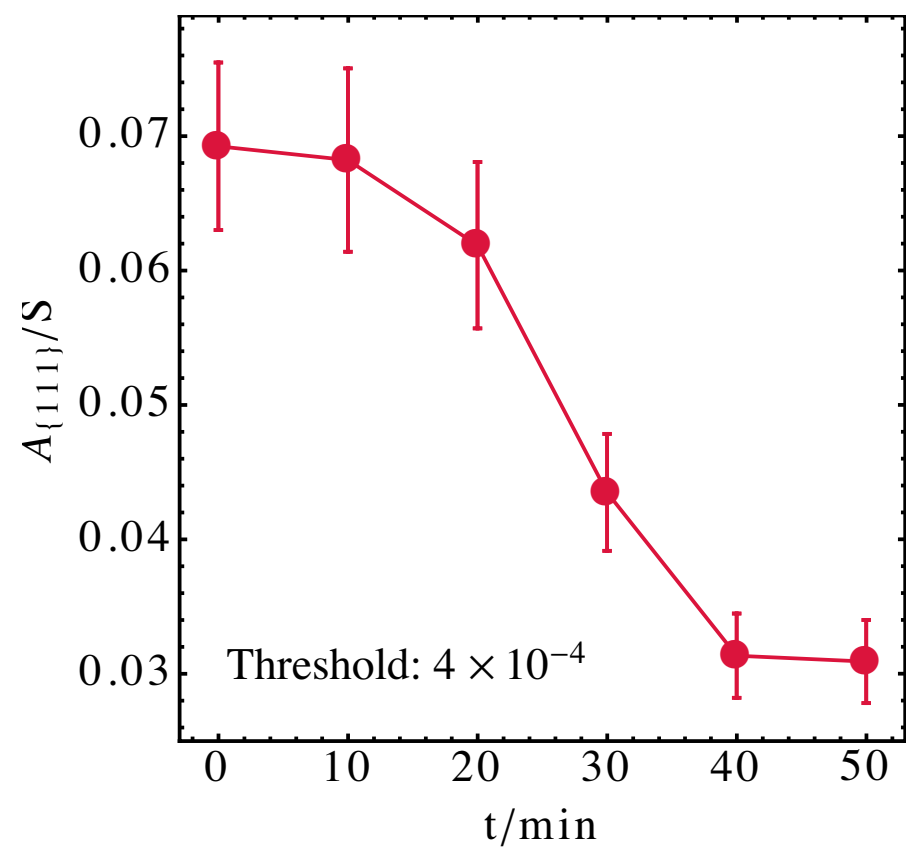

Figure 7: Area of $\{111\}$ patches in connected component $A B C D, A_{\{111\}}$, over total surface area, $S$, versus coarsening time. Fractional areas, $A_{\{111\}} / S$, were determined by summing over bins in the IND which had values above the threshold of $4 \times 10^{-4}$. Errors bars are due to binned patches that do not belong to connected component $A B C D$, but rather to similarly-oriented particles (roughly 5-8 \% error). The trend indicates that the Si particles evolve away from their equilibrium Wulff shape. 

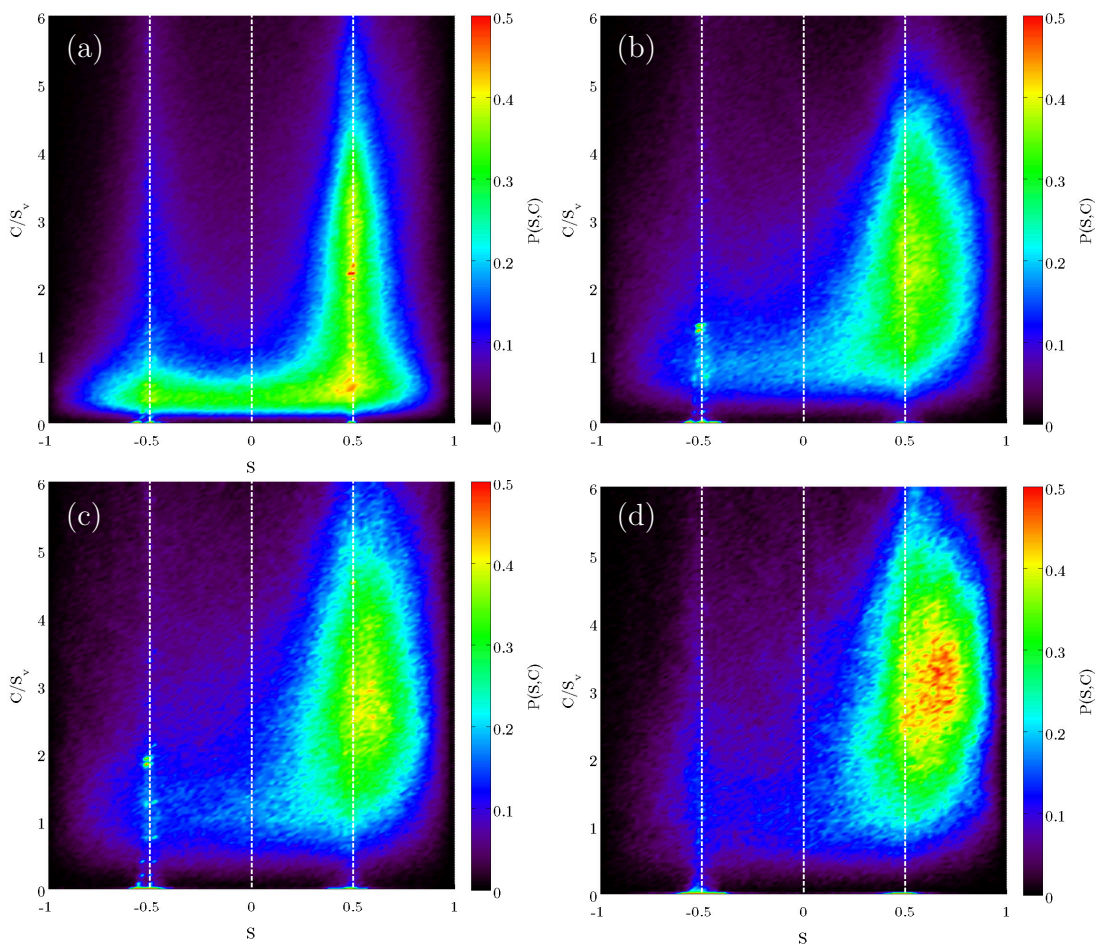

Figure 8: Interface shape distributions (ISDs) using the alternative $S$ versus $C$ classification [54], for the same microstructures shown in Fig. 2: (a) $t=0 \mathrm{~min}$. and $S_{v}^{-1}=19 \mu \mathrm{m}$, (b) $t=10 \mathrm{~min}$. and $S_{v}^{-1}=31 \mu \mathrm{m}$, (c) $t=20 \mathrm{~min}$. and $S_{v}^{-1}=42 \mu \mathrm{m}$, and (d) $t=50 \mathrm{~min}$. and $S_{v}^{-1}=53 \mu \mathrm{m}$. Scaling the curvedness, $C$, by $S_{v}$ and fixing the range of the color bar allow multiple coarsening times to be compared. Dashed lines at $S=\{-0.5,0,0.5\}$ serve as markers for liquid cylindrical-like shapes ("ruts"), saddle-like shapes, and solid cylindrical-like shapes ("ridges"), respectively. 

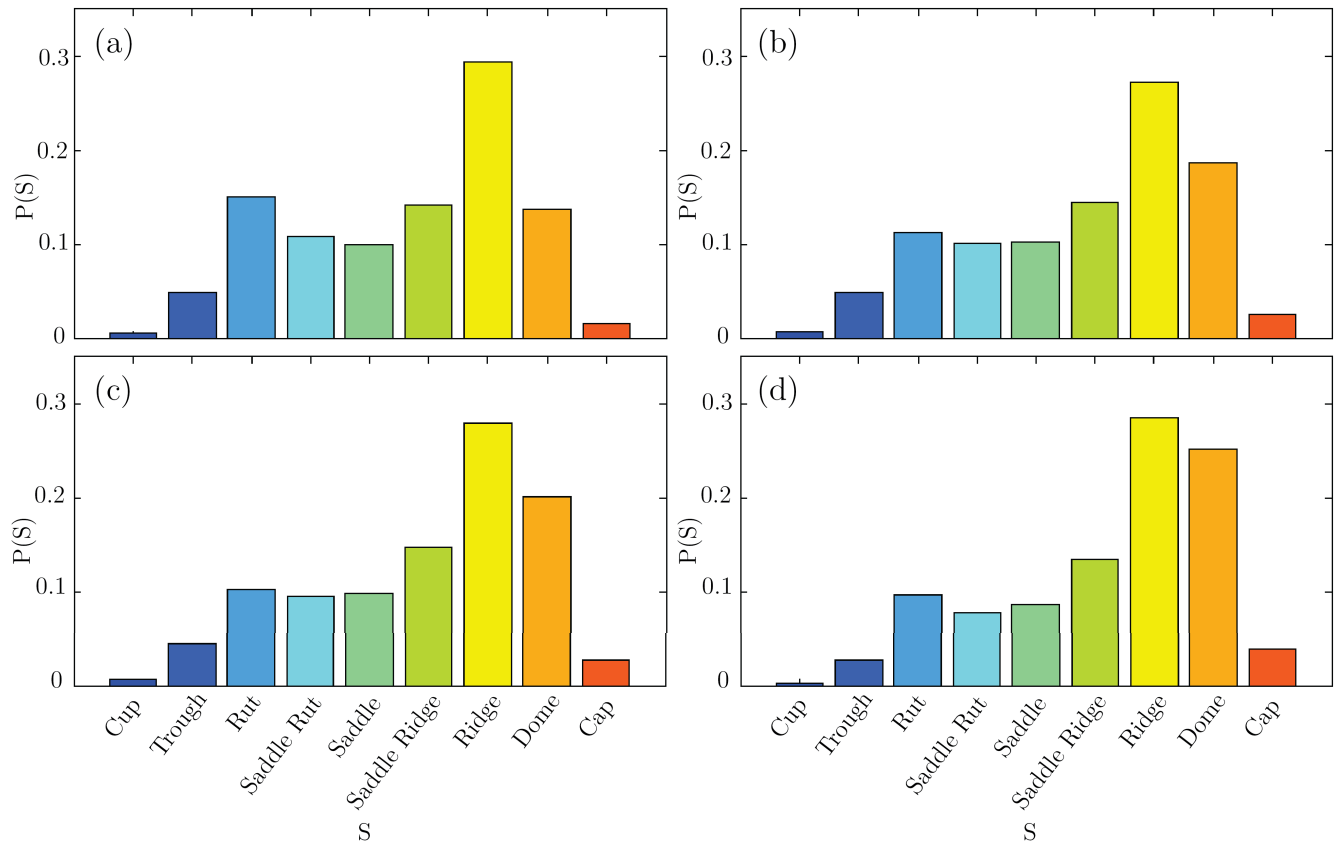

Figure 9: The probability of finding a patch of a given shape as a function of time, where (a) is $0 \mathrm{~min}$., (b) is 10 min., (c) is 20 min., and (d) is 50 min., of elapsed coarsening time. These plots are for the same microstructures measured in Fig. 8. We use the shape index scale of Fig. 1. The fractions of domes and spherical caps increase with time, suggesting that the coarsening evolution of primary Si particles is not self-similar. 

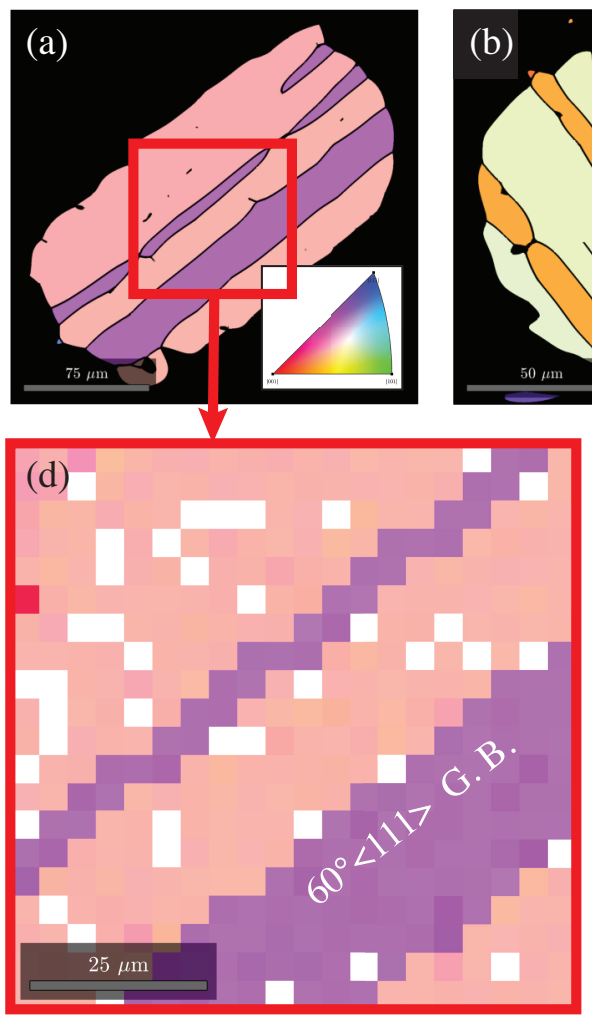
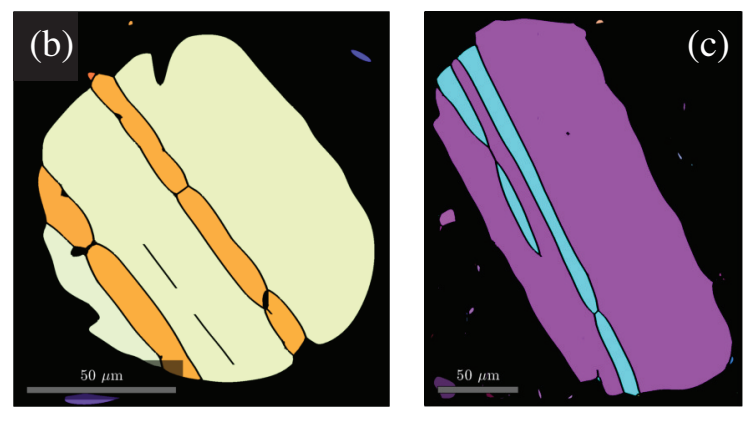

(e)

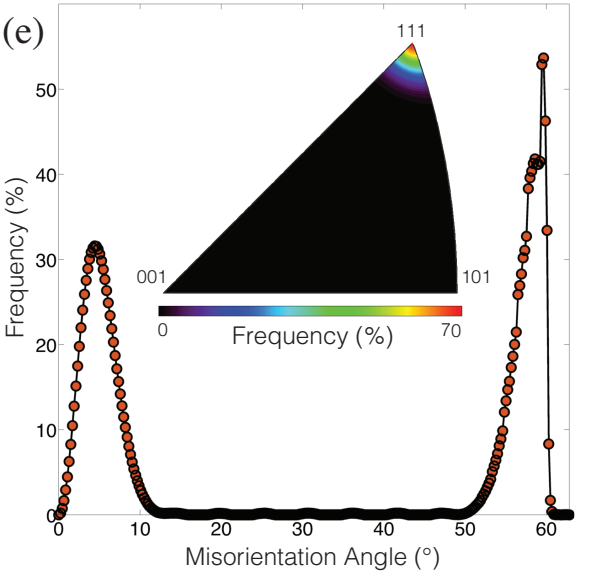

Figure 10: (a-c) Orientation maps of three representative Si particles that have coarsened for two days at $600^{\circ} \mathrm{C}$. The mean crystallographic orientation of each grain of $\mathrm{Si}$ is colored according to the standard stereographic triangle on the bottom-right of (a), while non-indexed regions belonging to the eutectic are colored black. (d) Discretized EBSD data of boxed region, where intra-granular variations and grain boundaries are apparent, and from which the uncorrelated misorientation distribution function (MODF) is calculated. The resulting MODF can then be represented as (e) misorientation angle and axis (inset) distributions. 


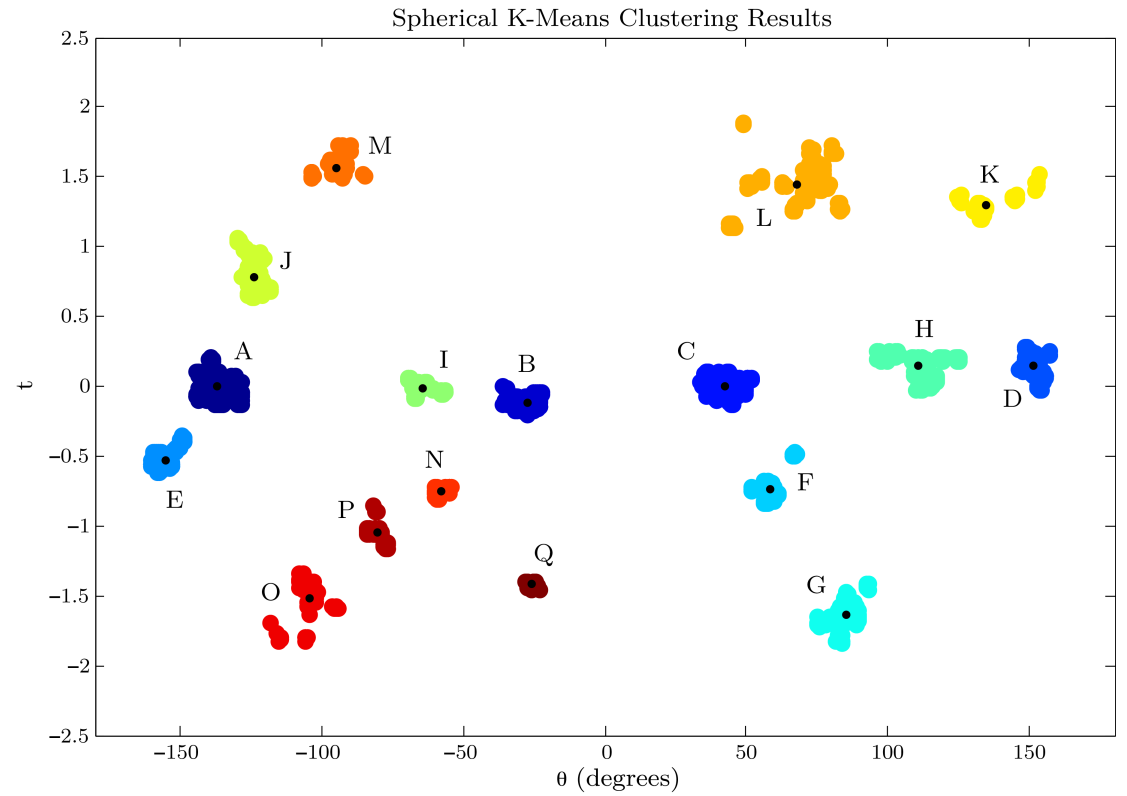

Figure 11: Results of spherical k-means (SKM) clustering of the interface normals from the as-cast microstructure. Different colors indicate different clusters; black points are the locations of the centroid vectors, $\{h k l\}$. We identify a total of 17 clusters, labelled $A$ through $Q$. 


\section{List of Tables}

$1 \quad$ Misorientation angles, $\alpha_{i j}$, between centroid vectors $i$ and $j$, where $i$ and $j \in\{A, Q\}$. Angles that match any of $70.53^{\circ}, 109.77^{\circ}$, or $180^{\circ}$ within an error of $10 \%$ are highlighted in red. These matches are then accepted or rejected (diagonal strikeout) as $\{111\}$ planes by examining the real-space reconstructions. . . . . . . . . . . . . . . . . 


\begin{tabular}{|c|c|c|c|c|c|c|c|c|c|c|c|c|c|c|c|c|c|}
\hline & A & B & $\mathrm{C}$ & $\mathrm{D}$ & $\mathrm{E}$ & $\mathrm{F}$ & G & $\mathrm{H}$ & I & $\mathrm{J}$ & $\mathrm{K}$ & $\mathrm{L}$ & M & $\mathrm{N}$ & $\mathrm{O}$ & $\mathrm{P}$ & $\mathrm{Q}$ \\
\hline $\mathrm{A}$ & 0.0 & 109.3 & 179.0 & 72.0 & 33.4 & 138.4 & 105.2 & 112.4 & 72.6 & 43.1 & 89.5 & 114.4 & 73.1 & 81.5 & 69.0 & 69.3 & 99.0 \\
\hline B & 109.3 & 0.0 & 69.7 & 177.9 & 118.7 & 82.2 & 91.9 & 138.3 & 37.3 & 99.1 & 125.3 & 98.3 & 87.5 & 42.6 & 78.5 & 62.3 & 56.2 \\
\hline $\mathrm{C}$ & 179.0 & 69.7 & 0.0 & 109.0 & 146.9 & 41.7 & 74.1 & 68.6 & 106.4 & 136.8 & 91.2 & 66.0 & 106.8 & 97.6 & 110.5 & 110.0 & 80.4 \\
\hline $\mathrm{D}$ & 72.0 & 177.9 & 109.0 & 0.0 & 63.3 & 97.9 & 89.4 & 40.4 & 143.7 & 80.6 & 52.7 & 79.6 & 91.3 & 139.6 & 103.6 & 119.9 & 125.6 \\
\hline $\mathrm{E}$ & 33.4 & 118.7 & 146.9 & 63.3 & 0.0 & 105.6 & 73.6 & 97.5 & 90.6 & 75.7 & 105.2 & 135.7 & 105.6 & 77.7 & 48.2 & 58.8 & 80.0 \\
\hline $\mathrm{F}$ & 138.4 & 82.2 & 41.7 & 97.9 & 105.6 & 0.0 & 32.6 & 67.8 & 114.0 & 177.5 & 116.8 & 102.5 & 148.4 & 81.9 & 74.9 & 83.0 & 53.5 \\
\hline $\mathrm{G}$ & 105.2 & 91.9 & 74.1 & 89.4 & 73.6 & 32.6 & 0.0 & 78.4 & 108.0 & 149.0 & 132.7 & 131.9 & 178.6 & 68.9 & 46.5 & 60.5 & 40.2 \\
\hline $\mathrm{H}$ & 112.4 & 138.3 & 68.6 & 40.4 & 97.5 & 67.8 & 78.4 & 0.0 & 170.9 & 110.0 & 54.5 & 63.0 & 102.9 & 147.0 & 117.9 & 136.2 & 117.1 \\
\hline I & 72.6 & 37.3 & 106.4 & 143.7 & 90.6 & 114.0 & 108.0 & 170.9 & 0.0 & 68.0 & 119.3 & 108.3 & 70.7 & 39.2 & 70.7 & 52.1 & 68.2 \\
\hline $\mathrm{J}$ & 43.1 & 99.1 & 136.8 & 80.6 & 75.7 & 177.5 & 149.0 & 110.0 & 68.0 & 0.0 & 60.6 & 75.2 & 30.0 & 100.5 & 107.5 & 99.5 & 129.1 \\
\hline $\mathrm{K}$ & 89.5 & 125.3 & 91.2 & 52.7 & 105.2 & 116.8 & 132.7 & 54.5 & 119.3 & 60.6 & 0.0 & 30.7 & 48.6 & 158.4 & 153.2 & 158.5 & 170.3 \\
\hline $\mathrm{L}$ & 114.4 & 98.3 & 66.0 & 79.6 & 135.7 & 102.5 & 131.9 & 63.0 & 108.2 & 75.2 & 30.7 & 0.0 & 49.5 & 140.3 & 176.1 & 159.3 & 144.0 \\
\hline $\mathrm{M}$ & 73.1 & 87.5 & 106.8 & 91.3 & 105.6 & 148.4 & 178.6 & 102.9 & 70.7 & 30.0 & 48.6 & 49.5 & 0.0 & 109.8 & 132.1 & 118.1 & 138.7 \\
\hline $\mathrm{N}$ & 81.5 & 42.6 & 97.6 & 139.6 & 77.7 & 81.9 & 68.9 & 147.0 & 39.2 & 100.5 & 158.4 & 140.3 & 109.8 & 0.0 & 37.0 & 19.7 & 30.0 \\
\hline $\mathrm{O}$ & 69.0 & 78.5 & 110.5 & 103.6 & 48.2 & 74.9 & 46.5 & 117.9 & 70.7 & 107.5 & 153.2 & 176.1 & 132.1 & 37.0 & 0.0 & 18.8 & 32.1 \\
\hline $\mathrm{P}$ & 69.2 & 62.3 & 110.0 & 119.9 & 58.8 & 83.0 & 60.5 & 136.2 & 52.1 & 99.5 & 158.5 & 159.3 & 118.1 & 19.7 & 18.8 & 0.0 & 30.8 \\
\hline Q & 99.0 & 56.2 & 80.4 & 125.6 & 80.0 & 53.5 & 40.2 & 117.1 & 68.2 & 129.1 & 170.3 & 144.0 & 138.7 & 30.0 & 32.1 & 30.8 & 0.0 \\
\hline
\end{tabular}

Table 1: Misorientation angles, $\alpha_{i j}$, between centroid vectors $i$ and $j$, where $i$ and $j \in\{A, Q\}$. Angles that match any of $70.53^{\circ}, 109.77^{\circ}$, or $180^{\circ}$ within an error of $10 \%$ are highlighted in red. These matches are then accepted or rejected (diagonal strikeout) as $\{111\}$ planes by examining the real-space reconstructions. 